Supporting Information for

\title{
Alkenes from Terminal Epoxides using Lithium 2,2,6,6-Tetramethylpiperidide and Organolithiums or Grignard Reagents
}

\author{
David M. Hodgson, ${ }^{\dagger}{ }^{\dagger}$ Matthew J. Fleming ${ }^{\dagger}$ and Steven J. Stanway ${ }^{\ddagger}$ \\ ${ }^{\dagger}$ Department of Chemistry, University of Oxford, Chemistry Research Laboratory, Mansfield Road, \\ Oxford OX1 3TA, U.K. \\ ${ }^{\ddagger}$ GlaxoSmithKline, New Frontiers Science Park, Third Avenue, Harlow, Essex, CM19 5AW, U.K.
}

General Directions All reactions required anhydrous conditions and were conducted in flame-dried glassware under an atmosphere of argon. Syringes and needles for the transfer of reagents were oven-dried and allowed to cool in a desiccator over self-indicating silica gel. All solvents were distilled under an atmosphere of argon; ethers from sodium benzophenone ketyl and hydrocarbons from $\mathrm{CaH}_{2}$. 2,2,6,6-Tetramethylpiperidine (TMP) and 1,2-epoxydodecane were distilled from $\mathrm{CaH}_{2}$ prior to use. Petrol refers to the fraction of light petroleum boiling at $30-40{ }^{\circ} \mathrm{C}$. Reactions were monitored by thin layer chromatography using commercially available aluminium backed plates pre-coated with silica containing a fluorescent indicator $\left(0.2 \mathrm{~mm}\right.$, Merck $\left.60 \mathrm{~F}_{254}\right)$ which were developed using phosphomolybdic acid solution. Column chromatography was performed on silica gel (Kieselgel 60, 40-63 $\mu \mathrm{m}$ ) or octadecyl-functionalized $\left(\mathrm{C}_{18}\right)$ silica gel. Infra-red spectra were obtained with liquid films of the compounds on $\mathrm{NaCl}$ discs on a PerkinElmer Paragon 1750 FTIR spectrophotometer. Peak intensities are specified as strong $(\mathrm{s})$, medium $(\mathrm{m})$, weak $(\mathrm{w})$ or broad $(\mathrm{br})$. Only selected absorbencies $\left(\mathrm{v}_{\max }\right)$ are reported. ${ }^{1} \mathrm{H}$ and ${ }^{13} \mathrm{C}$ NMR spectra were recorded in $\mathrm{CDCl}_{3}$ at $25{ }^{\circ} \mathrm{C}$, with Bruker DPX400, AV400 or AMX500 spectrometers. Chemical shifts are reported relative to $\mathrm{CHCl}_{3}$ for ${ }^{1} \mathrm{H}$ NMR (7.27 ppm) and $\mathrm{CDCl}_{3}$ for ${ }^{13} \mathrm{C}$ NMR (77.0 ppm). Coupling constants $(J)$ are given in $\mathrm{Hz}$, multiplicities are given as broad singlet (br), multiplet $(m)$, singlet (s), doublet (d), triplet (t), quartet (q), quintet (quint.) and septet (sept.). GCMS analyses were preformed on a Micromass GCT spectrometer with BPX5 column - HP 6890 (dimethylsilicon capillary column, $30 \mathrm{~m}, 0.25 \mathrm{~mm}$ i.d.) equipped with a mass selective detector operating at $60 \mathrm{eV}(\mathrm{Cl})$. Flow rate $(\mathrm{He})=1 \mathrm{~mL} / \mathrm{min}$. Mass spectra were obtained using a Micromass GCT spectrometer or by the EPSRC National Mass Spectrometry Service Centre at the University of Swansea using a $900 \mathrm{XLT}$ high resolution double focusing mass spectrometer with tandem Ion Trap. Solutions of commercially available and prepared organolithiums and 
Grignard reagents were titrated prior to use using $s-\mathrm{BuOH}$ and 1,10phenanthroline. ${ }^{1}$

\section{Procedures and Spectroscopic Data}

\section{General procedure A: for alkene formation using preformed or commercially available organolithiums, described for:}

(E)-1-Phenyl-1-dodecene ${ }^{2} \mathbf{6 a}$

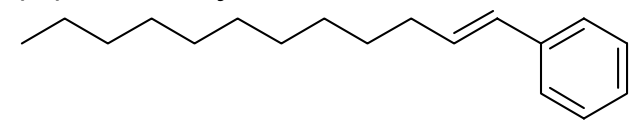

To a solution of 2,2,6,6-tetramethylpiperidine $(232 \mathrm{mg}, 1.64 \mathrm{mmol})$ in hexane (12 $\mathrm{mL}$ ) at $0{ }^{\circ} \mathrm{C}$ (ice bath) was added $n$-BuLi (1.6 M in hexanes; $1.03 \mathrm{~mL}, 1.64 \mathrm{mmol}$ ) dropwise. The reaction mixture was stirred at this temperature for $15 \mathrm{~min}$ and then a solution of $\mathrm{PhLi}\left(2.0 \mathrm{M}\right.$ in $\mathrm{Bu}_{2} \mathrm{O} ; 0.53 \mathrm{~mL}, 1.07 \mathrm{mmol}$ ) was added dropwise. The reaction was stirred for a further $5 \mathrm{~min}$ and then 1,2-epoxydodecane (151 $\mathrm{mg}, 0.82$ $\mathrm{mmol}$ ) was added. The ice bath was removed and the reaction mixture was left stirring for $2 \mathrm{~h}$. After quenching with saturated brine solution $(10 \mathrm{~mL})$, the layers were separated. The aqueous layer was extracted with $\mathrm{Et}_{2} \mathrm{O}(2 \times 10 \mathrm{~mL})$, the combined organic layers were dried $\left(\mathrm{MgSO}_{4}\right)$ and solvent removed in vacuo. The residue was purified by column chromatography $\left(\mathrm{SiO}_{2}\right.$, petrol) to give $(E)$-alkene $6 \mathbf{a}$ $\left(200 \mathrm{mg}, 93 \%, E: Z=98: 2\right.$, by GCMS analysis, $\mathrm{t}_{R}(Z)-6 \mathbf{a}=13.02 \mathrm{~min}, \mathrm{t}_{R}(E)-6 \mathbf{a}=$ 14.22 min initial temp. $=80^{\circ} \mathrm{C}$, max. temp. $=280{ }^{\circ} \mathrm{C}$, rate $=10^{\circ} \mathrm{C} / \mathrm{min}$ ) as a colourless oil; $\mathrm{R}_{\mathrm{f}} 0.48\left(\mathrm{SiO}_{2}\right.$, petrol). IR (neat)/cm ${ }^{-1} 3025 \mathrm{~m}, 2956 \mathrm{~s}, 2925 \mathrm{~s}, 2853 \mathrm{~s}$, 1625w, 1599w, 1494w, 1466m, 927s; ${ }^{1} \mathrm{H}$ NMR (400 MHz) $\delta 7.38-7.29(\mathrm{~m}, 4 \mathrm{H}), 7.21$ (tt, $J=7.3,1.5,1 \mathrm{H}), 6.41(\mathrm{~d}, J=15.9,1 \mathrm{H}), 6.26(\mathrm{dt}, J=15.9,6.8,1 \mathrm{H}), 2.22(\mathrm{dt}, J=$ $6.8,6.8,2 \mathrm{H}), 1.53-1.45(\mathrm{~m}, 2 \mathrm{H}), 1.40-1.25(\mathrm{~m}, 14 \mathrm{H}), 0.92(\mathrm{t}, J=6.7,3 \mathrm{H}) ;{ }^{13} \mathrm{C} N M R$ $(100 \mathrm{MHz}) \delta 138.0,131.3,129.7,128.5,126.7,125.9,33.1,32.0,29.7,29.6,29.4$, 29.4, 29.3, 22.7, 14.1; MS m/z (Cl) $245\left(\mathrm{M}+\mathrm{H}^{+}, 18\right), 244$ (57), 117 (47), 104 (100), 91 (14); HRMS calcd for $\mathrm{C}_{18} \mathrm{H}_{29}\left(\mathrm{M}+\mathrm{H}^{+}\right)$245.2269; found 245.2267.

\footnotetext{
${ }^{1}$ Schlosser, M. Organometallics in Synthesis, 2nd ed. Wiley, New York, 2002, p. 671.

2 Terao, J.; Watabe, H.; Miyamoto, M.; Kambe, N. Bull. Chem. Soc. Jpn. 2003, 76, 2209-2214.
} 
(E)-1,3-Tetradecadiene ${ }^{3}$ 6c

Following general procedure $A$, the addition of 1,2-epoxydodecane (100 $\mathrm{mg}, 0.54$ $\mathrm{mmol})$ to a solution of LTMP $(1.08 \mathrm{mmol})$ in hexane $(8 \mathrm{~mL})$ and vinyllithium ${ }^{4}(1.5 \mathrm{M}$ in $\left.\mathrm{Et}_{2} \mathrm{O} ; 0.54 \mathrm{~mL}, 0.81 \mathrm{mmol}\right)$ gave after workup and column chromatography $\left(\mathrm{SiO}_{2}\right.$, petrol) $(E)$-diene $6 \mathrm{c}\left(77 \mathrm{mg}, 73 \%, E: Z=98: 2\right.$ by GCMS analysis, $\mathrm{t}_{R}(Z)-6=6.83 \mathrm{~min}$, $\mathrm{t}_{R}(E)-6 \mathrm{c}=7.05 \mathrm{~min}$, initial temp. $=80^{\circ} \mathrm{C}$, max. temp. $=280^{\circ} \mathrm{C}$, rate $\left.=20{ }^{\circ} \mathrm{C} / \mathrm{min}\right)$ as a colourless oil; $\mathrm{R}_{\mathrm{f}} 0.58$ ( $\mathrm{SiO}_{2}$, petrol). IR (neat, $\left.\mathrm{cm}^{-1}\right)$ 3008m, 2925s, 2855s, 1794w, $1654 \mathrm{~m}, 1603 \mathrm{~m}, 1466 \mathrm{~s}, 1378 \mathrm{w}, 1001 \mathrm{~s}, 949 \mathrm{~m}, 895 \mathrm{~s} ;{ }^{1} \mathrm{H}$ NMR (400 MHz) $\delta 6.33$ (ddd, $J=16.9,10.2,10.2,1 \mathrm{H}), 6.06(\mathrm{dd}, J=15.1,10.2,1 \mathrm{H}), 5.72(\mathrm{dt}, J=15.1,7.1,1 \mathrm{H})$, $5.10(\mathrm{~d}, J=16.9,1 \mathrm{H}), 4.96(\mathrm{~d}, J=10.2,1 \mathrm{H}), 2.08(\mathrm{dt}, J=7.1,7.1,2 \mathrm{H}), 1.40-1.22$ $(\mathrm{m}, 16 \mathrm{H}), 0.90(\mathrm{t}, J=6.7,3 \mathrm{H}) ;{ }^{13} \mathrm{C} \operatorname{NMR}(100 \mathrm{MHz}) \delta 137.4,135.6,130.8,114.5$,

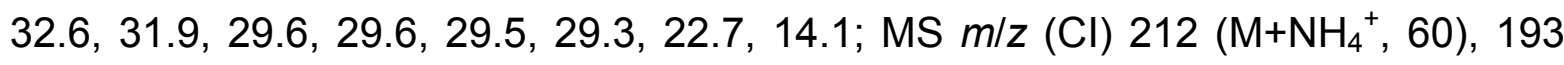
(9), 195 (100), 123 (8), 109 (13), 95 (28), 68 (9); HRMS calcd for $\mathrm{C}_{14} \mathrm{H}_{30} \mathrm{~N}\left(\mathrm{M}+\mathrm{NH}_{4}{ }^{+}\right)$ 212.2378, found 212.2379.

\section{$(2 E, 4 E)-2,4-P e n t a d e c a d i e n e ~ 6 e$}

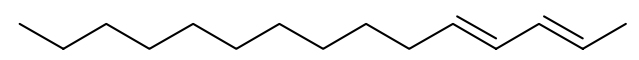

Following general procedure A, the addition of 1,2-epoxydodecane (100 $\mathrm{mg}, 0.54$ $\mathrm{mmol})$ to a solution of LTMP $(1.08 \mathrm{mmol})$ in hexane $(8 \mathrm{~mL})$ and $(E)$-propenyllithium ${ }^{5}$ (2.0 $\mathrm{M}$ in $\mathrm{Et}_{2} \mathrm{O} ; 0.35 \mathrm{~mL}, 0.70 \mathrm{mmol}$ ) gave after workup and column chromatography $\left(\mathrm{SiO}_{2}\right.$, petrol) $(E, E)$-diene $6 \mathrm{e}\left(92 \mathrm{mg}, 82 \%, E E: Z E=98: 2\right.$ by GCMS analysis, $\mathrm{t}_{R}$ $(Z, E)-6 \mathbf{e}=10.82 \mathrm{~min}, \mathrm{t}_{R}(E, E)-6 \mathbf{e}=10.98 \mathrm{~min}$, initial temp. $=100{ }^{\circ} \mathrm{C}$, max. temp. $=$

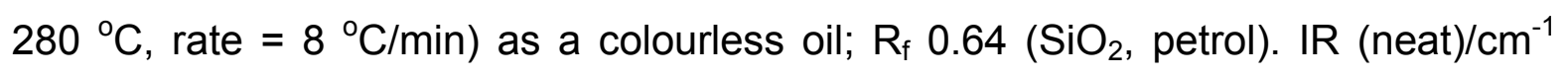
3016m, 2957s, 2925s, 2854s, 1466m, 1378w, 986s; ${ }^{1} \mathrm{H}$ NMR (400 MHz) $\delta 6.07-5.95$ $(\mathrm{m}, 2 \mathrm{H}), 5.62-5.52(\mathrm{~m}, 2 \mathrm{H}), 2.05(\mathrm{dt}, J=7.1,7.1,2 \mathrm{H}), 1.74(\mathrm{~d}, J=6.6,3 \mathrm{H}), 1.40-$ $1.22(\mathrm{~m}, 16 \mathrm{H}), 0.89(\mathrm{t}, J=6.8,3 \mathrm{H}) ;{ }^{13} \mathrm{C} \operatorname{NMR}(100 \mathrm{MHz}) \delta 132.3,131.7,130.2$, 126.6, 32.6, 31.9, 29.6, 29.5, 29.4, 29.3, 29.2, 22.7, 18.0, 14.1; MS m/z (Cl) 226 $\left(\mathrm{M}+\mathrm{NH}_{4}{ }^{+}, 33\right), 208$ (100), 207 (17), 95 (22), 81 (48); HRMS calcd for $\mathrm{C}_{15} \mathrm{H}_{32} \mathrm{~N}$ $\left(\mathrm{M}+\mathrm{NH}_{4}^{+}\right)$226.2535, found 226.2535 .

\footnotetext{
${ }^{3}$ Suzuki, K.; Hasegawa, T.; Imai, T.; Maeta, H.; Ohba, S. Tetrahedron 1995, 51, 4483-4494.

${ }^{4}$ Seyferth, D.; Weiner, M. A. J. Am. Chem. Soc. 1961, 83, 3583-3586.

5 Seyferth, D.; Vaughan, L. G. J. Am. Chem. Soc. 1964, 86, 883-890.
} 


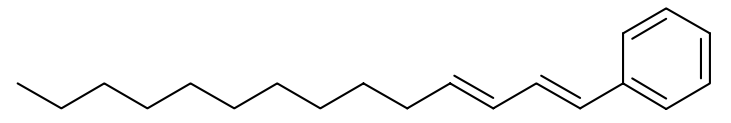

Following general procedure $\mathrm{A}$, the addition of 1,2-epoxydodecane (230 mg, 1.25 mmol) to a solution of LTMP $(2.50 \mathrm{mmol})$ in hexane $(19 \mathrm{~mL})$ and $(E)-2$ -

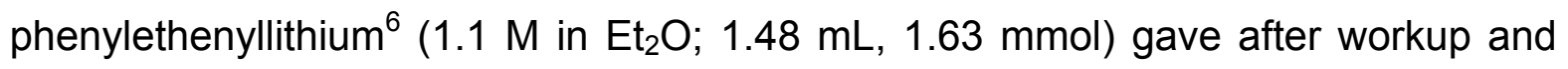
reverse phase column chromatography $\left(\mathrm{C}_{18} \text { silica, } \mathrm{MeCN}\right)^{7}(E, E)$-diene $\mathbf{6 g}(243 \mathrm{mg}$, $72 \%, E E: Z E=98: 2$, by GCMS analysis, $\mathrm{t}_{R}(Z, E)-\mathbf{6 g}=11.55 \mathrm{~min}, \mathrm{t}_{R}(E, E)-\mathbf{6 g}=11.83$ min, initial temp. $=80{ }^{\circ} \mathrm{C}$, max. temp. $=280^{\circ} \mathrm{C}$, rate $=20^{\circ} \mathrm{C} / \mathrm{min}$ ) as a colourless oil; $\mathrm{R}_{\mathrm{f}} 0.26$ ( $\mathrm{C}_{18}$ silica, MeCN). IR (neat)/cm $\mathrm{cm}^{-1} 3022 \mathrm{~m}, 2955 \mathrm{~s}, 2925 \mathrm{~s}, 2853 \mathrm{~s}, 1644 \mathrm{w}$, 1597w, 1495w, 1466w, 1448w, 986s; ${ }^{1} \mathrm{H}$ NMR (400 MHz) $\delta$ 7.40-7.37 (m, 2H), 7.33$7.28(\mathrm{~m}, 2 \mathrm{H}), 7.20(\mathrm{tt}, \mathrm{J}=7.3,1.3,1 \mathrm{H}), 6.76(\mathrm{dd}, J=15.7,10.4,1 \mathrm{H}), 6.44(\mathrm{~d}, J=$ 15.7, 1H), 6.20 (dd, $J=14.9,10.4,1 \mathrm{H}$ ), 5.83 (dt, $J=14.9,6.8,1 \mathrm{H}), 2.14(\mathrm{dt}, J=6.8$, $6.8,1 \mathrm{H}), 1.47-1.22(\mathrm{~m}, 16 \mathrm{H}), 0.88(\mathrm{t}, J=6.8,3 \mathrm{H}) ;{ }^{13} \mathrm{C}$ NMR $(100 \mathrm{MHz}) \delta 137.7$, 136.1, 130.4, 129.9, 129.5, 128.5, 127.0, 126.1, 32.9, 31.9, 29.6, 29.5, 29.4, 29.3, 29.2, 22.7, 14.1; MS m/z (Cl) $271\left(\mathrm{M}+\mathrm{H}^{+}\right.$100), 270 (64), 143 (25), 128 (14); HRMS calcd for $\mathrm{C}_{20} \mathrm{H}_{31}\left(\mathrm{M}+\mathrm{H}^{+}\right)$271.2426; found 271.2429.

\section{$(2 Z, 4 E)-2,4-P e n t a d e c a d i e n e$ 6h}

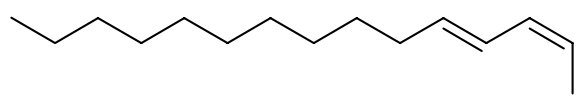

Following general procedure $A$, the addition of 1,2-epoxydodecane (100 mg, 0.54 $\mathrm{mmol})$ to a solution of LTMP $(1.08 \mathrm{mmol})$ in hexane $(8 \mathrm{~mL})$ and $(Z)$-propenyllithium ${ }^{5}$ (1.4 $\mathrm{M}$ in $\mathrm{Et}_{2} \mathrm{O} ; 0.50 \mathrm{~mL}, 0.70 \mathrm{mmol}$ ) gave after workup and column chromatography $\left(\mathrm{SiO}_{2}\right.$, petrol) $(Z, E)$-diene-6h $\left(79 \mathrm{mg}, 70 \%, Z E: E E=90: 10\right.$ by GCMS analysis, $\mathrm{t}_{R}$ $(Z, E)-6 \mathbf{h}=10.82 \mathrm{~min}, \mathrm{t}_{R}(E, E)-6 \mathbf{h}=10.98 \mathrm{~min}$, initial temp. $=100{ }^{\circ} \mathrm{C}$, max. temp. $=$ $280{ }^{\circ} \mathrm{C}$, rate $\left.=8{ }^{\circ} \mathrm{C} / \mathrm{min}\right)$ as a colourless oil; $\mathrm{R}_{\mathrm{f}} 0.64\left(\mathrm{SiO}_{2}\right.$, petrol $)$. IR (neat)/cm ${ }^{-1} 3015 \mathrm{~m}, 2960 \mathrm{~s}, 2920 \mathrm{~s}, 2844 \mathrm{~s}, 1466 \mathrm{~m}, 1349 \mathrm{w}, 970 \mathrm{~s} ;{ }^{1} \mathrm{H}$ NMR (400 $\mathrm{MHz}) \delta 6.33(\mathrm{ddq}, J=10.9,10.1,1.5,1 \mathrm{H}), 6.02-5.95(\mathrm{~m}, 1 \mathrm{H}), 5.67(\mathrm{dt}, J=14.9,7.3$, $1 \mathrm{H}$ ), 5.38 (dq, $J=10.9,7.1,1 \mathrm{H}), 2.15-2.07(\mathrm{~m}, 2 \mathrm{H}), 1.75(\mathrm{dd}, J=7.1,1.5,3 \mathrm{H}), 1.44-$ $1.20(\mathrm{~m}, 16 \mathrm{H}), 0.89(\mathrm{t}, J=6.8,3 \mathrm{H}) ;{ }^{13} \mathrm{C}$ NMR $(100 \mathrm{MHz}) \delta 134.7,129.5,125.3$, 123.8, 32.9, 31.9, 29.6, 29.5, 29.4, 29.4, 29.3, 22.7, 14.1, 13.3; MS MS m/z (Cl) 226

\footnotetext{
${ }^{6}$ Prepared by the addition of $(E)-\beta$-bromostyrene to Li metal in $\mathrm{Et}_{2} \mathrm{O}$ at $0{ }^{\circ} \mathrm{C}$.

${ }^{7}$ Forina, V. J. Org. Chem. 1991, 56, 4985-4987.
} 
$\left(\mathrm{M}+\mathrm{NH}_{4}{ }^{+}, 52\right), 208$ (100), 207 (11), 166 (87), 95 (10), 81 (22); HRMS calcd for $\mathrm{C}_{15} \mathrm{H}_{32} \mathrm{~N}\left(\mathrm{M}+\mathrm{NH}_{4}{ }^{+}\right)$226.2535; found 226.2541.

(E)-1-Trimethylsilyl-2-tridecene ${ }^{8} \mathbf{7 a}$

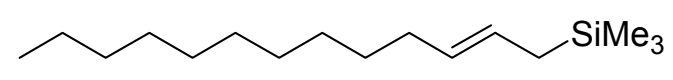

Following general procedure $A$, the addition of 1,2-epoxydodecane (100 mg, 0.54 $\mathrm{mmol})$ to a solution of LTMP $(0.81 \mathrm{mmol})$ in $\mathrm{Et}_{2} \mathrm{O}(8 \mathrm{~mL})$ and (trimethylsilylmethyl)lithium $(1.0 \mathrm{M}$ in pentane; $0.81 \mathrm{~mL}, 0.81 \mathrm{mmol}$ ) gave after workup and column chromatography $\left(\mathrm{SiO}_{2}, 100 \%\right.$ petrol) $(E)$-allylsilane $7 \mathrm{a}(89 \mathrm{mg}$, $65 \%, E: Z=97: 3$, by GCMS analysis, $\mathrm{t}_{R}(E)-7 \mathrm{a}=8.22 \mathrm{~min}, \mathrm{t}_{R}(Z)-7 \mathrm{a}=8.30 \mathrm{~min}$, initial temp. $=80{ }^{\circ} \mathrm{C}$, max. temp. $=280{ }^{\circ} \mathrm{C}$, rate $=20{ }^{\circ} \mathrm{C} / \mathrm{min}$ ) as a colourless oil; $R_{f}$ $0.63\left(\mathrm{SiO}_{2}\right.$, petrol). IR (neat) $/ \mathrm{cm}^{-1} 3007 \mathrm{~m}, 2928 \mathrm{~s}, 2854 \mathrm{~s}, 1659 \mathrm{w}, 1466 \mathrm{~s}, 1416 \mathrm{~m}$, $1378 \mathrm{w}, 1248 \mathrm{~s}, 1155 \mathrm{~s}, 934 \mathrm{~s}, 857 \mathrm{~s} ;{ }^{1} \mathrm{H}$ NMR $(400 \mathrm{MHz}) \delta 5.42-5.33(\mathrm{~m}, 1 \mathrm{H}), 5.29-$ $5.23(\mathrm{~m}, 1 \mathrm{H}), 2.29(\mathrm{dt}, J=6.8,6.8,2 \mathrm{H}), 1.41(\mathrm{dd}, J=7.6,1.0,2 \mathrm{H}), 1.33-1.27(\mathrm{~m}$, $16 \mathrm{H}), 0.90(\mathrm{t}, J=6.8,3 \mathrm{H}), 0.00(\mathrm{~s}, 9 \mathrm{H}) ;{ }^{13} \mathrm{C}$ NMR $(100 \mathrm{MHz}) \delta 129.1,125.9,32.8$, 31.9, 30.0, 29.7, 29.6, 29.5, 29.4, 29.1, 22.7, 22.6, 14.1, -2.0; MS m/z (Cl) 255 $\left(\mathrm{M}+\mathrm{H}^{+}, 25\right), 90$ (100), 73(10); HRMS calcd for $\mathrm{C}_{16} \mathrm{H}_{36} \mathrm{Si}\left(\mathrm{M}+\mathrm{H}^{+}\right)$255.2508; found 255.2501.

(E)-(4-Methylpent-2-enyl)-trimethylsilane ${ }^{9} \mathbf{7 b}$

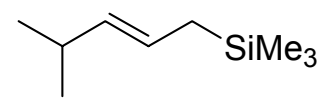

Following general procedure A, the addition of 3-methyl-1,2-epoxybutane (123 mg, $1.43 \mathrm{mmol})$ to a solution of LTMP $(2.15 \mathrm{mmol})$ in THF $(21 \mathrm{~mL})$ and (trimethylsilylmethyl)lithium (1.0 M in pentane; $2.15 \mathrm{~mL}, 2.15 \mathrm{mmol}$ ) gave after workup and column chromatography $\left(\mathrm{SiO}_{2}\right.$, petrol) $(E)$-allylsilane $\mathbf{7 b}(159 \mathrm{mg}, 71 \%$, $E: Z=98: 2$, by GCMS analysis, $t_{R}(E)-7 \mathbf{b}=3.15 \mathrm{~min}, \mathrm{t}_{R}(Z)-7 \mathbf{b}=3.23$, min initial temp. $=60{ }^{\circ} \mathrm{C}$, max. temp. $=240{ }^{\circ} \mathrm{C}$, rate $=20^{\circ} \mathrm{C} / \mathrm{min}$ ) as a colourless oil; $R_{\mathrm{f}} 0.66$ ( $\mathrm{SiO}_{2}$, petrol). IR (neat)/cm ${ }^{-1} 2957 \mathrm{~s}, 2926 \mathrm{~s}, 2871 \mathrm{~m}, 1466 \mathrm{w}, 1248 \mathrm{~s}, 859 \mathrm{~s}, 840 \mathrm{~s} ;{ }^{1} \mathrm{H}$ NMR $(400 \mathrm{MHz}) \delta 5.39-5.30(\mathrm{~m}, 1 \mathrm{H}), 5.23(\mathrm{dd}, J=15.2,6.8,1 \mathrm{H}), 2.24$ (d,sept, $J=$ $6.8,6.8,1 \mathrm{H}), 1.39(\mathrm{~d}, J=7.6,1 \mathrm{H}), 0.97(\mathrm{~d}, J=6.8,6 \mathrm{H}), 0.00(\mathrm{~s}, 9 \mathrm{H}) ;{ }^{13} \mathrm{C} \operatorname{NMR}(100$

\footnotetext{
${ }^{8}$ Masao, A.; Yamaguchi, H.; Fujita, E.; Nagao, Y.; Ochiai, M. Chem. Pharm. Bull. 1989, 37, 3221 3324.

${ }^{9}$ Shimizu, N.; Imazu, S.; Shibata, F.; Tsuno, Y. Bull. Chem. Soc. Jpn. 1991, 64, 1122-1128.
} 
$\mathrm{MHz}) \delta$ 136.4, 122.8, 31.3, 23.0, 22.4, -2.1; MS m/z (Cl) $157\left(\mathrm{M}+\mathrm{H}^{+}, 12\right), 91$ (12), 90 (100); HRMS calcd for $\mathrm{C}_{9} \mathrm{H}_{22} \mathrm{Si}\left(\mathrm{M}+\mathrm{H}^{+}\right)$157.1413; found 157.1416.

(E)-(4,4-Dimethyl-pent-2-enyl)-trimethylsilane ${ }^{9}$ 7c<smiles>C[AsH3]CC=CC(C)(C)C</smiles>

Following general procedure $A$, the addition of dimethyl 1,2-epoxybutane (123 mg, $1.23 \mathrm{mmol})$ to a solution of LTMP $(1.85 \mathrm{mmol})$ in THF $(18 \mathrm{~mL})$ and (trimethylsilylmethyl)lithium (1.0 $\mathrm{M}$ in pentane; $1.85 \mathrm{~mL}, 1.85 \mathrm{mmol}$ ) gave after workup and column chromatography $\left(\mathrm{SiO}_{2}\right.$, petrol) $(E)$-allylsilane $7 \mathrm{c}(132 \mathrm{mg}, 63 \%$, E-only by GCMS analysis, $\mathrm{t}_{R}(E)-7 \mathrm{c}=4.33 \mathrm{~min}$, initial temp. $=50{ }^{\circ} \mathrm{C}$, max. temp. $=$ $240{ }^{\circ} \mathrm{C}$, rate $\left.=20{ }^{\circ} \mathrm{C} / \mathrm{min}\right)$ as a colourless oil; $\mathrm{R}_{\mathrm{f}} 0.63\left(\mathrm{SiO}_{2}\right.$, petrol $)$. IR (neat) $/ \mathrm{cm}^{-1} 2925 \mathrm{~s}, 2854 \mathrm{~s}, 2361 \mathrm{w}, 1464 \mathrm{w}, 1378 \mathrm{~m}, 1248 \mathrm{w}, 1156 \mathrm{~m}, 969 \mathrm{~m}, 851 \mathrm{~m}$; ${ }^{1} \mathrm{H}$ NMR $(400 \mathrm{MHz}) \delta 5.30-5.25(\mathrm{~m}, 2 \mathrm{H}), 1.39(\mathrm{dd}, J=4.8,2.0,2 \mathrm{H}), 0.99(\mathrm{~s}, 9 \mathrm{H})$, -0.01 (s, 9H); ${ }^{13} \mathrm{C}$ NMR $(100 \mathrm{MHz}) \delta 140.2,120.4,32.9,30.0,22.4,-2.1 ; \mathrm{MS} \mathrm{m} / \mathrm{z}$ (Cl) $171\left(\mathrm{M}+\mathrm{H}^{+}, 27\right), 155$ (9), 148 (10), 91 (12), 90 (100), 73 (23); HRMS calcd for $\mathrm{C}_{10} \mathrm{H}_{23} \mathrm{Si}\left(\mathrm{M}+\mathrm{H}^{+}\right)$171.1569; found 171.1561.

\section{General Procedure B: for alkene formation using in-situ formation of organolithium, described for:}

(E)-1-Dodec-1-enyl-4-methoxy-benzene 6b

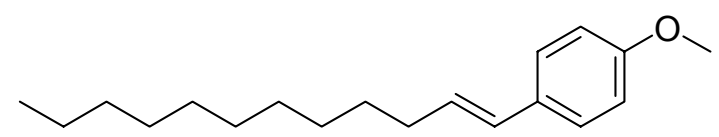

To a solution of 4-bromoanisole $(131 \mathrm{mg}, 0.70 \mathrm{mmol})$ in THF $(1.5 \mathrm{~mL})$ at $-78{ }^{\circ} \mathrm{C}$ was added $t$-BuLi (1.5 M in pentane; $0.94 \mathrm{~mL}, 1.40 \mathrm{mmol}$ ) dropwise. The reaction mixture was stirred at this temperature for $1 \mathrm{~h}$ and then warmed to $0{ }^{\circ} \mathrm{C}$ (ice bath). After 5 min a solution of LTMP (prepared by the addition of $n$-BuLi (1.6 M in hexanes; $0.68 \mathrm{~mL}, 1.08 \mathrm{mmol}$ ) to 2,2,6,6-tetramethylpiperidine (153 mg, $1.08 \mathrm{mmol}$ ) in hexane $(8 \mathrm{~mL})$ at $\left.0{ }^{\circ} \mathrm{C}\right)$ was added dropwise. The reaction was left for a further 5 min at $0{ }^{\circ} \mathrm{C}$ and then 1,2-epoxydodecane (100 $\mathrm{mg}, 0.54 \mathrm{mmol}$ ) was added. The ice bath was removed and the reaction mixture was stirred for a further $2 \mathrm{~h}$. After quenching with saturated brine solution $(10 \mathrm{~mL})$, the layers were separated. The aqueous layer was extracted with $\mathrm{Et}_{2} \mathrm{O}(2 \times 10 \mathrm{~mL})$, the combined organic layers 
were dried $\left(\mathrm{MgSO}_{4}\right)$ and solvent evaporated in vacuo. The residue was purified by column chromatography $\left(\mathrm{SiO}_{2}, 2 \% \mathrm{Et}_{2} \mathrm{O}\right.$ in petrol) to give $(E)$-alkene $\mathbf{6 b}(104 \mathrm{mg}$, $70 \%, E: Z=98: 2$ by GCMS analysis, $t_{R}(Z)-6 \mathbf{b}=16.18 \mathrm{~min}, \mathrm{t}_{R}(E)-6 \mathbf{b}=16.78 \mathrm{~min}$, initial temp. $=100{ }^{\circ} \mathrm{C}$, max. temp. $=280{ }^{\circ} \mathrm{C}$, rate $=10{ }^{\circ} \mathrm{C} / \mathrm{min}$ ) as a white solid; $R_{f}$ $0.48\left(\mathrm{SiO}_{2}, 10 \% \mathrm{Et}_{2} \mathrm{O}\right.$ in petrol $), \mathrm{mp}=36-39{ }^{\circ} \mathrm{C}$. IR $\left(\mathrm{CHCl}_{3}\right) / \mathrm{cm}^{-1} 2954 \mathrm{~m}, 2920 \mathrm{~s}$, $2850 \mathrm{~m}, 1608 \mathrm{w}, 1513 \mathrm{w}, 1465 \mathrm{w}, 1251 \mathrm{w}, 1176 \mathrm{w}, 1023 \mathrm{w}, 962 \mathrm{w} ;{ }^{1} \mathrm{H}$ NMR $(400 \mathrm{MHz}) \delta$ $7.31(\mathrm{~d}, J=8.8,2 \mathrm{H}), 6.87$ (d, $J=8.8,2 \mathrm{H}), 6.35$ (d, $J=15.7,1 \mathrm{H}), 6.12$ (dt, $J=15.7$, 7.1, 1H), $3.82(\mathrm{~s}, 3 \mathrm{H}), 2.21(\mathrm{dt}, J=7.1,7.1,2 \mathrm{H}), 1.52-1.44(\mathrm{~m}, 2 \mathrm{H}), 1.41-1.25(\mathrm{~m}$, $14 \mathrm{H}), 0.92$ (t, $J=6.9,3 \mathrm{H}) ;{ }^{13} \mathrm{C} \mathrm{NMR}(100 \mathrm{MHz}) \delta 158.6,130.8,129.1,129.0,127.0$, 113.9, 55.3, 33.1, 32.0, 29.7, 29.6, 29.6, 29.4, 29.3, 22.7, 14.1; MS m/z (Cl) 275 $\left(\mathrm{M}+\mathrm{H}^{+}, 100\right), 274$ (69), 147 (63), 134 (18), 121 (25); HRMS calcd for $\mathrm{C}_{19} \mathrm{H}_{31} \mathrm{O}$ $\left(\mathrm{M}+\mathrm{H}^{+}\right)$275.2375; found 275.2373 .

(E)-2,3-Dimethyl-2,4-pentadecadiene 6d

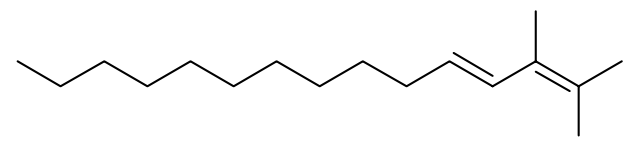

Following general procedure B, 2-bromo-3-methyl-2-butene (105 mg, $0.70 \mathrm{mmol})$, $t$ BuLi (1.5 M in pentane; $0.94 \mathrm{~mL}, 1.40 \mathrm{mmol})$ in THF $(1.5 \mathrm{~mL})$ then a solution of LTMP $(1.08 \mathrm{mmol})$ in hexane $(8 \mathrm{~mL})$ and then 1,2-epoxydodecane $(100 \mathrm{mg}, 0.54$ mmol) gave after workup and column chromatography $\left(\mathrm{SiO}_{2}\right.$, petrol) $(E)$-diene $\mathbf{6 d}$ (109 mg, 85\%, E-only by GCMS analysis, $\mathrm{t}_{R}(E)-6 \mathbf{d}=7.10 \mathrm{~min}$, initial temp. $=80^{\circ} \mathrm{C}$, max. temp. $=280{ }^{\circ} \mathrm{C}$, rate $\left.=20^{\circ} \mathrm{C} / \mathrm{min}\right)$ as a colourless oil; $\mathrm{R}_{\mathrm{f}} 0.56\left(\mathrm{SiO}_{2}\right.$, petrol $)$. IR (neat) $/ \mathrm{cm}^{-1}$ 2923s, 2854s, 1465m, 1375m, 1170w, 959s; ${ }^{1} \mathrm{H}$ NMR $(400 \mathrm{MHz}) \delta 6.05$ (d, $J=15.4,1 \mathrm{H}), 5.59(\mathrm{dt}, J=15.4,7.1,1 \mathrm{H}), 2.12(\mathrm{dt}, J=7.0,7.0,2 \mathrm{H}), 1.82(\mathrm{~s}, 3 \mathrm{H})$, $1.78(\mathrm{~s}, 3 \mathrm{H}), 1.76(\mathrm{~s}, 3 \mathrm{H}), 1.33-1.24(\mathrm{~m}, 16 \mathrm{H}), 0.89(\mathrm{t}, J=6.8,3 \mathrm{H}) ;{ }^{13} \mathrm{C}$ NMR $(100$ $\mathrm{MHz}) \delta 129.1,128.5,128.4$ 126.1, 33.4, 31.9, 30.0, 29.6, 29.6, 29.4, 29.3, 22.7, 21.6, 20.2, 14.4, 14.1; MS m/z (Cl) $237\left(\mathrm{M}+\mathrm{H}^{+}, 100\right), 109$ (21); HRMS calcd for $\mathrm{C}_{17} \mathrm{H}_{33}\left(\mathrm{M}+\mathrm{H}^{+}\right)$, 237.2582; found 237.2585 . 
$(7 E, 9 E)-7,9-$ Icosadiene $6 f$

Following general procedure $\mathrm{B},(E)$-1-iodo-1-octene ${ }^{10}(134 \mathrm{mg}, 0.56 \mathrm{mmol}), t$-BuLi $(1.5 \mathrm{M}$ in pentane; $0.74 \mathrm{~mL}, 1.12 \mathrm{mmol})$ in $\mathrm{Et}_{2} \mathrm{O}(1.0 \mathrm{~mL})$ then a solution of LTMP $(0.87 \mathrm{mmol})$ in hexane $(7 \mathrm{~mL})$ and then 1,2-epoxydodecane $(80 \mathrm{mg}, 0.43 \mathrm{mmol})$ gave after workup and column chromatography $\left(\mathrm{SiO}_{2}\right.$, petrol) $(E, E)$-diene $6 \mathbf{f}$ (101 $\mathrm{mg}, 84 \%, E E: Z E=99: 1$, by GCMS analysis, $\mathrm{t}_{R}(Z, E)-6 \mathbf{f}=16.68 \mathrm{~min}, \mathrm{t}_{R}(E, E)-6 \mathbf{f}=$ $17.07 \mathrm{~min}$, initial temp. $=80{ }^{\circ} \mathrm{C}$, max. temp. $=280{ }^{\circ} \mathrm{C}$, rate $=10{ }^{\circ} \mathrm{C} / \mathrm{min}$ ) as a colourless oil; $\mathrm{R}_{\mathrm{f}} 0.59\left(\mathrm{SiO}_{2}\right.$, petrol). IR (neat)/cm $\mathrm{cm}^{-1} 3014 \mathrm{w}, 29557 \mathrm{~s}, 2925 \mathrm{~s}, 2854 \mathrm{~s}$, 1460m, 1378w, 986s; ${ }^{1} \mathrm{H}$ NMR (400 MHz) $\delta 6.03-5.98(\mathrm{~m}, 2 \mathrm{H}), 5.61-5.53(\mathrm{~m}, 2 \mathrm{H})$, 2.05 (dt, $J=7.1,7.1,4 \mathrm{H}), 1.42-1.21(\mathrm{~m}, 24 \mathrm{H}), 0.89(\mathrm{t}, J=6.8,6 \mathrm{H}) ;{ }^{13} \mathrm{C}$ NMR $(100$ $\mathrm{MHz}) \delta 132.4,130.3,32.6,31.9,31.7,29.6,29.5,29.4,29.4,29.3,29.2,22.7,22.6$,

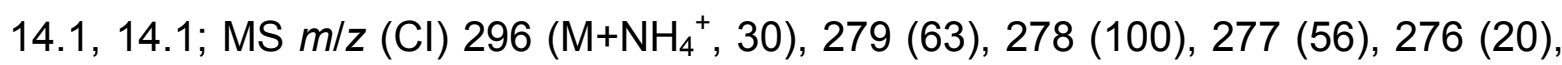
275 (17), 138 (13), 95 (16), 81 (15); HRMS calcd for $\mathrm{C}_{20} \mathrm{H}_{42} \mathrm{~N}\left(\mathrm{M}+\mathrm{NH}_{4}{ }^{+}\right)$296.3317; found 296.3310 .

$(7 Z, 9 E)-7,9-$ Icosadiene ${ }^{11} \mathbf{6 i}$

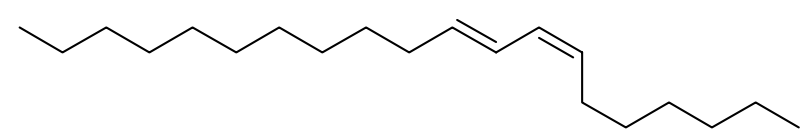

Following general procedure $\mathrm{B},(Z)-1$-iodo-1-octene ${ }^{12}(134 \mathrm{mg}, 0.56 \mathrm{mmol}), t$-BuLi $(1.5 \mathrm{M}$ in pentane; $0.74 \mathrm{~mL}, 1.12 \mathrm{mmol})$ in $\mathrm{Et}_{2} \mathrm{O}(1.0 \mathrm{~mL})$ then a solution of LTMP $(0.87 \mathrm{mmol})$ in hexane $(7 \mathrm{~mL})$ and then 1,2-epoxydodecane $(80 \mathrm{mg}, 0.43 \mathrm{mmol})$ gave after workup and column chromatography $\left(\mathrm{SiO}_{2}\right.$, petrol) $(Z, E)$-diene $6 \mathbf{i}(96 \mathrm{mg}$, $80 \%, Z E: E E$ :other isomer $=91: 5: 4$ by GCMS analysis, $\mathrm{t}_{R}(Z, E)-6 \mathbf{i}=16.68 \mathrm{~min}, \mathrm{t}_{R}$ other isomer $=16.90, \mathrm{t}_{R}(E, E)-6 \mathbf{i}=17.07 \mathrm{~min}$, initial temp. $=80^{\circ} \mathrm{C}$, max. temp. $=$ $280{ }^{\circ} \mathrm{C}$, rate $=10{ }^{\circ} \mathrm{C} / \mathrm{min}$ ) as a colourless oil; $\mathrm{R}_{\mathrm{f}} 0.59\left(\mathrm{SiO}_{2}\right.$, petrol). IR (neat) $/ \mathrm{cm}^{-1}$ $3019 \mathrm{~m}, 2926 \mathrm{~s}, 2854 \mathrm{~s}, 1466 \mathrm{~s}, 1378 \mathrm{~s}, 982 \mathrm{~m}, 946 \mathrm{~m}$; ${ }^{1} \mathrm{H}$ NMR $(400 \mathrm{MHz}) \delta 6.35-6.26$ $(\mathrm{m}, 1 \mathrm{H}), 6.03-5.92(\mathrm{~m}, 1 \mathrm{H}), 5.67(\mathrm{dt}, J=15.2,7.3,1 \mathrm{H}), 5.31(\mathrm{dt}, J=10.6,7.6,1 \mathrm{H})$, 2.20-2.02 (m, 4H), 1.43-1.21 (m, 24H), $0.89(\mathrm{t}, J=6.8,3 \mathrm{H}) ;{ }^{13} \mathrm{C} \mathrm{NMR}(100 \mathrm{MHz}) \delta$ 134.7, 130.1, 128.6, 125.6, 32.9, 31.9, 31.8, 29.7, 29.6, 29.5, 29.4, 29.3, 29.2, 28.9, 27.7, 22.7, 22.6, 14.1, 14.1; MS $\mathrm{m} / \mathrm{z}(\mathrm{Cl}) 296\left(\mathrm{M}+\mathrm{NH}_{4}{ }^{+}, 31\right), 279$ (75), 278 (100),

\footnotetext{
${ }^{10}$ Schwartz, J.; Hart, D. W.; Blackburn, T. F. J. Am. Chem. Soc. 1975, 97, 679-680.

${ }^{11}$ Bestmann, H. J.; Vostrowsky, O.; Paulus, H.; Billmann, W.; Stransky. W. Tetrahedron Lett. 1977, $18,121-124$.

${ }^{12}$ Brown, H. C.; Blue, C. D.; Nelson, D. J.; Bhat, N. G. J. Org. Chem. 1989, 54, 6064-6067.
} 
277 (31), 276 (13), 166 (10), 138 (14), 96 (14), 81 (19); HRMS calcd for $\mathrm{C}_{20} \mathrm{H}_{42} \mathrm{~N}$ $\left(\mathrm{M}+\mathrm{NH}_{4}{ }^{+}\right)$296.3317; found 296.3325 .

(5E,7E)-5-Methyl-5,7-octadecadiene 6j

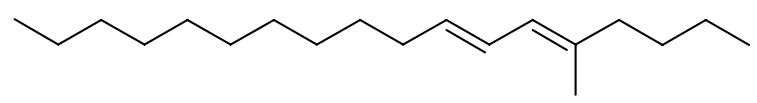

Following general procedure $\mathrm{B},(E)$-1-iodo-2-methyl-1-hexene ${ }^{13}$ (198 mg, 0.88 $\mathrm{mmol}), t$-BuLi (1.5 M in pentane; $1.18 \mathrm{~mL}, 1.77 \mathrm{mmol})$ in $\mathrm{Et}_{2} \mathrm{O}(2 \mathrm{~mL})$ then a solution of LTMP $(1.36 \mathrm{mmol})$ in hexane $(10 \mathrm{~mL})$ and then 1,2-epoxydodecane $(125 \mathrm{mg}$, $0.68 \mathrm{mmol})$ gave after workup and column chromatography $\left(\mathrm{SiO}_{2}, 100 \%\right.$ petrol) $(E, E)$-diene $6 \mathbf{j}\left(153 \mathrm{mg}, 85 \% E E: Z E=91: 9\right.$ by GCMS analysis, $\mathrm{t}_{R}(Z, E)-6 \mathbf{j}=9.78$ $\min , \mathrm{t}_{R}(E, E)-6 \mathbf{j}=10.00 \mathrm{~min}$, initial temp. $=80^{\circ} \mathrm{C}$, max. temp. $=280^{\circ} \mathrm{C}$, rate $=20$ ${ }^{\circ} \mathrm{C} / \mathrm{min}$ ) as a colourless oil; $\mathrm{R}_{\mathrm{f}} 0.69\left(\mathrm{SiO}_{2}\right.$, petrol). IR (neat)/cm ${ }^{-1} 3020 \mathrm{~m}, 2924 \mathrm{~s}$, 2854s, 1642w, 1466s, 1378m, 963s; ${ }^{1} \mathrm{H}$ NMR $(400 \mathrm{MHz}) \delta 6.25$ (dd, $J=14.9,10.9$, $1 \mathrm{H}$ ), 5.81 (d, $J=10.9,1 \mathrm{H}), 5.59$ (dt, $J=14.9,7.1,1 \mathrm{H}), 2.09$ (dt, $J=7.1,7.1,2 \mathrm{H}$ ), $2.04(\mathrm{t}, J=7.6,2 \mathrm{H}), 1.74(\mathrm{~s}, 3 \mathrm{H}), 1.44-1.26(\mathrm{~m}, 2 \mathrm{H}), 0.93-0.88(\mathrm{~m}, 6 \mathrm{H}) ;{ }^{13} \mathrm{C} N M R$ $(100 \mathrm{MHz}) \delta 136.7,132.4,126.6,124.5,39.5,33.0,31.9,30.1,29.6,29.6,29.4$, 29.3, 29.3, 22.7, 22.4, 16.4, 14.1, 14.0; MS m/z (Cl) $265\left(\mathrm{M}+\mathrm{H}^{+}, 100\right), 264$ (29), 263 (10), 137 (10); HRMS calcd for $\mathrm{C}_{19} \mathrm{H}_{37}\left(\mathrm{M}+\mathrm{H}^{+}\right)$265.2895; found 265.2889.

\section{General Procedure C: for allylsilane formation using in-situ formation of $\alpha$ - silylithium, described for:}

(E)-(1-Pentyltridec-2-en-1-yl)trimethylsilane 7d

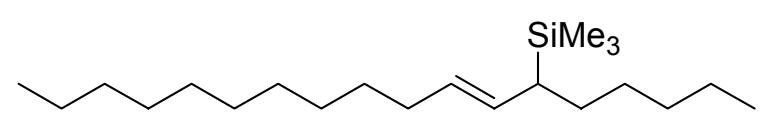

To a solution of vinyltrimethylsilane $(109 \mathrm{mg}, 1.09 \mathrm{mmol})$ in THF $(1 \mathrm{~mL})$ at $-78{ }^{\circ} \mathrm{C}$ was added $n$-BuLi (1.5 M in hexanes; $0.54 \mathrm{~mL}, 0.81 \mathrm{mmol}$ ) dropwise. The reaction mixture was stirred at this temperature for $2 \mathrm{~h}$ and then at $-30{ }^{\circ} \mathrm{C}$ for $2 \mathrm{~h}$. The reaction mixture was warmed to $0{ }^{\circ} \mathrm{C}$ (ice bath) and a solution of LTMP (prepared from the addition of $n$-BuLi (1.5 M in hexanes; $0.54 \mathrm{~mL}, 0.81 \mathrm{mmol}$ ) to 2,2,6,6tetramethylpiperidine $(114 \mathrm{mg}, 0.81 \mathrm{mmol})$ in hexane $(7 \mathrm{~mL})$ at $0{ }^{\circ} \mathrm{C}$ ) was added dropwise. After $5 \mathrm{~min}$ 1,2-epoxydodecane (100 mg, $0.54 \mathrm{mmol}$ ) was added and the reaction mixture was left stirring for $2 \mathrm{~h}$. After quenching with

\footnotetext{
${ }^{13}$ Negishi, E.; Van Horn, D. E.; Yoshida, T. J. Am. Chem. Soc. 1985, 107, 6639-6647.
} 
saturated brine solution $(10 \mathrm{~mL})$, the layers were separated. The aqueous layer was extracted with $\mathrm{Et}_{2} \mathrm{O}(2 \times 10 \mathrm{~mL})$, the combined organic layers were dried $\left(\mathrm{MgSO}_{4}\right)$ and solvent evaporated in vacuo. The residue was purified by reverse phase column chromatography $\left(\mathrm{C}_{18} \text { silica, } 15 \% \mathrm{Et}_{2} \mathrm{O} \text { in } \mathrm{MeCN}\right)^{7}$ to give $(E)$-allylsilane $7 \mathrm{~d}$ (130 $\mathrm{mg}$, $74 \%$, E-only by GCMS analysis, $(E)-7 d=10.18 \mathrm{~min}$, initial temp. $=80{ }^{\circ} \mathrm{C}$, $\max$. temp. $=280{ }^{\circ} \mathrm{C}$, rate $\left.=20{ }^{\circ} \mathrm{C} / \mathrm{min}\right)$ as a colourless oil; $\mathrm{R}_{\mathrm{f}} 0.13\left(\mathrm{C}_{18}\right.$ silica, $15 \% \mathrm{Et}_{2} \mathrm{O}$ in MeCN). IR (neat)/cm ${ }^{-1} 2924 \mathrm{~s}, 2854 \mathrm{~s}, 1467 \mathrm{~m}, 1378 \mathrm{w}, 1258 \mathrm{~m}, 969 \mathrm{~m}, 861 \mathrm{~m} ;{ }^{1} \mathrm{H}$ NMR $(400 \mathrm{MHz}) \delta 5.24-5.12(\mathrm{~m}, 2 \mathrm{H}), 1.99(\mathrm{dt}, J=6.5,6.5,2 \mathrm{H}), 1.42-1.16(\mathrm{~m}, 25 \mathrm{H}), 0.91-$ $0.86(\mathrm{~m}, 6 \mathrm{H}),-0.01(\mathrm{~s}, 9 \mathrm{H}) ;{ }^{13} \mathrm{C}$ NMR $(100 \mathrm{MHz}) \delta 131.5,128.2,32.9,32.9,31.9$, 31.8, 30.1, 29.7, 29.6, 29.5, 29.4, 29.1, 29.0, 28.9, 22.7, 22.6, 14.2, 14.1, -3.1; MS m/z (Cl) $325\left(\mathrm{M}+\mathrm{H}^{+}, 34\right), 251$ (13), (8), 250 (48), 90 (100), 73 (25); HRMS calcd for $\mathrm{C}_{21} \mathrm{H}_{45} \mathrm{Si}\left(\mathrm{M}+\mathrm{H}^{+}\right)$325.3291; found 325.3287.

(E)-(1-pentylundeca-2,10-dien-1-yl)trimethylsilane 7e

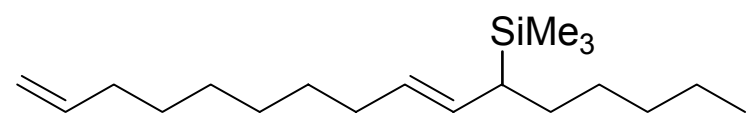

Following general procedure $\mathrm{C}$, the addition of 1,2-epoxy-9-decene (83 $\mathrm{mg}, 0.54$ $\mathrm{mmol}$ ) instead of 1,2-epoxydodecane gave after workup and reverse phase column chromatography $\left(\mathrm{C}_{18} \text { silica, MeCN }\right)^{7}(E)$-allylsilane $7 \mathrm{e}(120 \mathrm{mg}, 75 \%$, E-only by GCMS analysis, $(E)-7 e=11.57$ min, initial temp. $=80^{\circ} \mathrm{C}$, max. temp. $=280{ }^{\circ} \mathrm{C}$, rate $\left.=15^{\circ} \mathrm{C} / \mathrm{min}\right)$ as a colourless oil; $\mathrm{R}_{\mathrm{f}} 0.13\left(\mathrm{C}_{18}\right.$ silica, MeCN). IR (neat) $/ \mathrm{cm}^{-1} 3078 \mathrm{~s}$, 2926s, 2854s, 1642w, 1464w, 1247s, 836s; ${ }^{1} \mathrm{H}$ NMR (400 MHz) $\delta 5.87-5.77(\mathrm{~m}, 1 \mathrm{H})$, 5.24-5.12 (m, 2H), 5.03-4.92 (m, 2H), $2.05(\mathrm{dt}, J=6.8,6.8,2 \mathrm{H}), 1.99(\mathrm{dt}, J=6.5$, $6.5,2 \mathrm{H}), 1.44-1.14(\mathrm{~m}, 17 \mathrm{H}), 0.89(\mathrm{t}, \mathrm{J}=6.9,3 \mathrm{H}),-0.05(\mathrm{~s}, 9 \mathrm{H}) ;{ }^{13} \mathrm{C}$ NMR $(100 \mathrm{MHz})$ $\delta 139.3,131.5,128.1,114.1,33.8,32.9,32.8,31.8,30.0,29.0,28.9,28.9,28.8$, 22.7, 14.1, -3.2; MS m/z (Cl) $295\left(\mathrm{M}+\mathrm{H}^{+}, 40\right), 220$ (29), 90 (100); HRMS calcd for $\mathrm{C}_{19} \mathrm{H}_{39} \mathrm{Si}\left(\mathrm{M}+\mathrm{H}^{+}\right)$295.2821; found 295.2822. 


\section{S 11}

(E)-(1-pentyl-5-phenylpent-2-en-1-yl)trimethysilane 7f

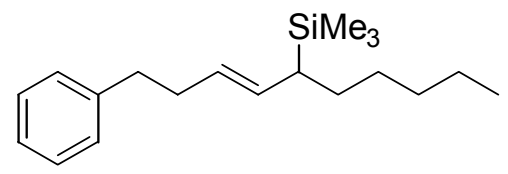

Following general procedure $C$, the addition of 1,2-epoxy-4-phenylbutane ${ }^{14}(80 \mathrm{mg}$, $0.54 \mathrm{mmol}$ ) instead of 1,2-epoxydodecane gave after workup and column chromatography $\left(\mathrm{SiO}_{2}\right.$, petrol) (E)-allylsilane $7 f(122 \mathrm{mg}, 78 \%, E: Z=98: 2$ by GCMS analysis, $\mathrm{t}_{R}(Z)-\mathbf{7 f}=12.33 \mathrm{~min}, \mathrm{t}_{R}(E)-7 \mathrm{f}=12.43 \mathrm{~min}$, initial temp. $=80{ }^{\circ} \mathrm{C}$, $\max$. temp. $=280{ }^{\circ} \mathrm{C}$, rate $\left.=15^{\circ} \mathrm{C} / \mathrm{min}\right)$ as a colourless oil; $\mathrm{R}_{\mathrm{f}} 0.48\left(\mathrm{SiO}_{2}\right.$, petrol $)$. IR (neat)/cm ${ }^{-1} 3064 \mathrm{w}, 3027 \mathrm{~m}, 2925 \mathrm{~s}, 2853 \mathrm{~s}, 1605 \mathrm{w}, 1496 \mathrm{~m}, 1454 \mathrm{~m}, 1247 \mathrm{~s} ;{ }^{1} \mathrm{H}$ NMR $(400 \mathrm{MHz}) \delta$ 7.37-7.28 (m, 2H), 7.24-7.18 (m, 3H), 5.31-5.20 (m, 2H), $2.70(\mathrm{t}, J=$ 7.3, 2H), 2.40 (dt, $J=7.3,7.3,2 \mathrm{H}), 1.47-1.13(\mathrm{~m}, 9 \mathrm{H}), 0.92(\mathrm{t}, J=7.1,3 \mathrm{H}$ ), -0.04 (s, $9 \mathrm{H}) ;{ }^{13} \mathrm{C}$ NMR $(100 \mathrm{MHz}) \delta 142.2,132.4,128.5,128.2,127.0,125.6,36.6,34.7$, 33.0, 31.8, 29.0, 28.8, 22.6, 14.2, -3.2; MS m/z (Cl) $289\left(\mathrm{M}+\mathrm{H}^{+}, 100\right) 215$ (19), 214 (91), 197 (29), 123 (23), 90 (40); HRMS calcd for $\mathrm{C}_{19} \mathrm{H}_{33} \mathrm{Si}\left(\mathrm{M}+\mathrm{H}^{+}\right)$289.2352; found 289.2344 .

(E)-1-(tert-Butyldimethylsilanyloxy)-6-(trimethylsilyl)undec-4-ene 7g

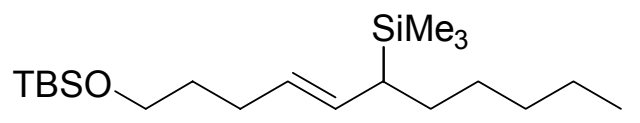

Following general procedure $\mathrm{C}$, the addition of 1-tert-butyldimethylsilyoxy-4,5epoxypentane $^{15}$ (117 mg, $0.54 \mathrm{mmol}$ ) instead of 1,2-epoxydodecane gave after workup and column chromatography $\left(\mathrm{SiO}_{2}\right.$, petrol) $(E)$-allylsilane $\mathbf{7 g}(138 \mathrm{mg}, 71 \%$, $E$-only by GCMS analysis, $(E)-\mathbf{7 g}=10.17 \mathrm{~min}$, initial temp. $=80^{\circ} \mathrm{C}$, max. temp. $=$ $280{ }^{\circ} \mathrm{C}$, rate $\left.=15{ }^{\circ} \mathrm{C} / \mathrm{min}\right)$ as a colourless oil; $\mathrm{R}_{\mathrm{f}} 0.20$ ( $\mathrm{SiO}_{2}$, petrol). IR (neat) $/ \mathrm{cm}^{-1} 2929 \mathrm{~m}, 2857 \mathrm{~m}, 1471 \mathrm{w}, 1388 \mathrm{w}, 1248 \mathrm{~m}, 1105 \mathrm{~m}, 969 \mathrm{w}, 836 \mathrm{~m} ;{ }^{1} \mathrm{H}$ $\operatorname{NMR}(400 \mathrm{MHz}) \delta 5.25-5.14(\mathrm{~m}, 2 \mathrm{H}), 3.62(\mathrm{t}, J=6.8,2 \mathrm{H}), 2.05(\mathrm{dt}, J=6.8,6.8,2 \mathrm{H})$, $1.58(\mathrm{dd}, J=6.8,6.8,2 \mathrm{H}), 1.47-1.12(\mathrm{~m}, 9 \mathrm{H}), 0.91(\mathrm{~s}, 9 \mathrm{H}), 0.88(\mathrm{t}, J=6.8,3 \mathrm{H}), 0.06$ $(\mathrm{s}, 6 \mathrm{H}),-0.05(\mathrm{~s}, 9 \mathrm{H}) ;{ }^{13} \mathrm{C}$ NMR $(100 \mathrm{MHz}) \delta 131.9,127.5,62.8,33.2,33.0,31.8$, 29.1, 29.0, 28.8, 26.0, 22.6, 18.4, 14.2, -3.1, -5.3; MS m/z (Cl) $357\left(\mathrm{M}+\mathrm{H}^{+}, 31\right), 299$ (32), 269 (21), 242 (16), 228 (19), 225 (77), 164 (43), 147 (100), 90 (46), 73 (55); HRMS calcd for $\mathrm{C}_{21} \mathrm{H}_{45} \mathrm{Si}\left(\mathrm{M}+\mathrm{H}^{+}\right)$357.3009; found 357.3011 .

\footnotetext{
${ }^{14}$ Elings, J. A.; Downing, R. S.; Sheldon, R. A. Eur. J. Org. Chem. 1999, 837-846.

${ }^{15}$ Yang, L.; Weber, A. E.; Greenlee, W. J.; Patchett, A. A. Tetrahedron Lett. 1993, 34, 7035-7038.
} 
General procedure A was used for alkene formation using deuterated epoxides cis and trans $8^{16}$ (Table 3), E:Z ratio was determined by GCMS analysis and the deuterium content at the vinylic position ( ${ }^{1} \mathrm{H}$ NMR analysis) determined by mass spectrometry.

\section{General Procedure D: for alkene formation using alkyl Grignard reagents and LTMP, described for:}

\section{(E)-5-Hexadecene $\mathbf{6 k}$}

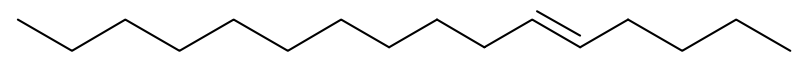

To a solution of 2,2,6,6-tetramethylpiperidine $(153 \mathrm{mg}, 1.08 \mathrm{mmol})$ in $\mathrm{Et}_{2} \mathrm{O}(8 \mathrm{~mL})$ at $0{ }^{\circ} \mathrm{C}$ (ice bath) was added $n$-BuLi (1.6 M in hexanes; $0.68 \mathrm{~mL}, 1.08 \mathrm{mmol}$ ) dropwise. The reaction mixture was stirred at this temperature for $15 \mathrm{~min}$ and then a solution of $\mathrm{BuMgCl}\left(2.0 \mathrm{M}\right.$ in $\mathrm{Et}_{2} \mathrm{O} ; 0.38 \mathrm{~mL}, 0.76 \mathrm{mmol}$ ) was added dropwise. The reaction was stirred for a further $5 \mathrm{~min}$ and then 1,2-epoxydodecane (100 $\mathrm{mg}, 0.54 \mathrm{mmol}$ ) was added. The ice bath was removed and the reaction mixture was left stirring for $3 \mathrm{~h}$. After quenching with saturated brine solution $(10 \mathrm{~mL})$, the layers were separated. The aqueous layer was extracted with $\mathrm{Et}_{2} \mathrm{O}(2 \times 10 \mathrm{~mL})$ and the combined organic layers were dried $\left(\mathrm{MgSO}_{4}\right)$ and solvent removed in vacuo. The residue was purified by column chromatography $\left(\mathrm{SiO}_{2}\right.$, petrol) to give $(E)$-alkene $\mathbf{6 k}$ (84 mg, $69 \%, E: Z=96: 4$, by ${ }^{1} \mathrm{H}$ NMR analysis of vinylic region) as a colourless oil; $\mathrm{R}_{\mathrm{f}} 0.70$ ( $\mathrm{SiO}_{2}$, petrol). IR (neat)/ $/ \mathrm{cm}^{-1} 2924 \mathrm{~s}, 2854 \mathrm{~s} 1466 \mathrm{~m}, 1378 \mathrm{w}, 966 \mathrm{~m} ;{ }^{1} \mathrm{H}$ NMR $(500 \mathrm{MHz}) \delta$ 5.43-5.83 (m, 2H), 2.03-1.93 (m, 4H), 1.39-1.22 (m, 20H), 0.93-0.87 $(\mathrm{m}, 6 \mathrm{H}) ;{ }^{13} \mathrm{C}$ NMR $(125 \mathrm{MHz}) \delta 130.3,130.2,32.6,32.2,31.9,31.8,29.6,29.5,29.3$, 29.1, 22.6, 22.1, 14.0, 13.9; MS m/z (EI) $224\left(\mathrm{M}^{+}, 21\right), 111$ (39), 98 (29), 97 (88), 84 (47), 84 (47), 83 (100), 71 (32), 70 (59), 69 (92), 67 (41), 57 (30), 55 (65); HRMS calcd for $\mathrm{C}_{16} \mathrm{H}_{32}\left(\mathrm{M}^{+}\right)$224.2504; found 224.2514 .

\section{(E)-2-Tridecene 6I}

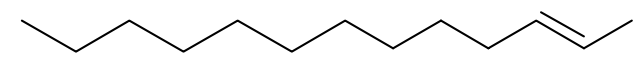

Following general procedure $\mathrm{D}$, the addition of 1,2-epoxydodecane (100 $\mathrm{mg}, 0.54$ $\mathrm{mmol})$ to a solution of LTMP $(0.81 \mathrm{mmol})$ in hexane $(8 \mathrm{~mL})$ and $\mathrm{MeMgCl}(2.2 \mathrm{M}$ in

${ }^{16}$ (a) Yanagisawa, A.; Yasue, K.; Yamamoto, H. J. Chem. Soc., Chem. Commun. 1994, 2103-2104;

(b) Patrick, D. W.; Truesdale, L. K.; Biller, S. A.; Sharpless, K. B. J. Org. Chem. 1978, 43, 2628-2638. 
THF; $0.45 \mathrm{~mL}, 0.98 \mathrm{mmol})$ gave after workup and column chromatography $\left(\mathrm{SiO}_{2}\right.$, petrol) $(E)$-alkene $6 \mathrm{I}\left(70 \mathrm{mg}, 71 \%, E: Z=98: 2\right.$ by GCMS analysis, $\mathrm{t}_{R}(E)-6 \mathrm{I}=9.10$ $\min , \mathrm{t}_{R}(Z)-6 \mathbf{I}=9.25 \mathrm{~min}$, initial temp. $=80{ }^{\circ} \mathrm{C}$, max. temp. $=280{ }^{\circ} \mathrm{C}$, rate $=10$ ${ }^{\circ} \mathrm{C} / \mathrm{min}$ ) as a colourless oil; $\mathrm{R}_{\mathrm{f}} 0.66\left(\mathrm{SiO}_{2}\right.$, petrol). IR (neat, $\left.\mathrm{cm}^{-1}\right) 2925 \mathrm{~s}, 2855 \mathrm{~s}$, 1465m, 1378w, 964m; ${ }^{1} \mathrm{H}$ NMR (400 MHz) $\delta 5.44-5.40(\mathrm{~m}, 2 \mathrm{H}), 1.99-1.94(\mathrm{~m}, 2 \mathrm{H})$, 1.66-1.64 (m, 3H), 1.35-1.24 (m, 16H), $0.89(\mathrm{t}, J=6.8,3 \mathrm{H}) ;{ }^{13} \mathrm{C}$ NMR $(100 \mathrm{MHz}) \delta$ 131.7, 124.5, 32.6, 31.9, 29.6, 29.5, 29.4, 29.2, 22.7, 17.9, 14.1; MS m/z (EI) 182 $\left(\mathrm{M}^{+}, 41\right), 111$ (32), 98 (34), 97 (87), 84 (61), 83 (94), 71 (37), 70 (83), 69 (100), 67 (38), 55 (71); HRMS calcd for $\mathrm{C}_{13} \mathrm{H}_{26}\left(\mathrm{M}^{+}\right)$182.2035, found 182.2035 .

\section{General Procedure E: for alkene formation using vinyl Grignard reagents and LTMP, described for:}

$(7 Z, 9 E)-7,9-$ Icosadiene ${ }^{10} \mathbf{6 i}$

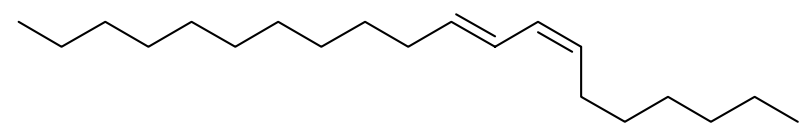

To a solution of (Z)-1-iodo-1-octene ${ }^{11}(181 \mathrm{mg}, 0.76 \mathrm{mmol})$ in THF $(2 \mathrm{~mL})$ at $-78{ }^{\circ} \mathrm{C}$ was added $t$-BuLi (1.5 M in pentane; $1.01 \mathrm{~mL}, 1.52 \mathrm{mmol}$ ) dropwise. The reaction mixture was stirred at this temperature for $1 \mathrm{~h}$ and then warmed to $0{ }^{\circ} \mathrm{C}$ (ice bath). Anhydrous $\mathrm{MgCl}_{2}$ (72 $\mathrm{mg}, 0.76 \mathrm{mmol}$ ) was added in one portion and the reaction mixture left stirring at $0{ }^{\circ} \mathrm{C}$ for $1 \mathrm{~h}$. A solution of LTMP (prepared by the addition of $n$ BuLi (1.6 M in hexanes; $0.68 \mathrm{~mL}, 1.08 \mathrm{mmol}$ ) to 2,2,6,6-tetramethylpiperidine (153 $\mathrm{mg}, 1.08 \mathrm{mmol})$ in $\mathrm{Et}_{2} \mathrm{O}(8 \mathrm{~mL})$ at $\left.0{ }^{\circ} \mathrm{C}\right)$ was added dropwise. The reaction was left for a further $5 \mathrm{~min}$ at $0{ }^{\circ} \mathrm{C}$ and then 1,2-epoxydodecane $(100 \mathrm{mg}, 0.54 \mathrm{mmol})$ was added. The ice bath was removed and the reaction mixture was stirred for a further $3 \mathrm{~h}$. After quenching with saturated brine solution $(10 \mathrm{~mL})$, the layers were separated. The aqueous layer was extracted with $\mathrm{Et}_{2} \mathrm{O}(3 \times 10 \mathrm{~mL})$, the combined organic layers were dried $\left(\mathrm{MgSO}_{4}\right)$ and solvent evaporated in vacuo. The residue was purified by column chromatography $\left(\mathrm{SiO}_{2}\right.$, petrol) to give $(Z, E)$-diene $\mathbf{6 i}$ (96 $\mathrm{mg}$, $67 \%, Z E: E E$ :other isomer $=91: 5: 4$ by GCMS analysis) as a colourless oil.

See above for data. 
(5E,7E)-5-Methyl-5,7-octadecadiene 6j

Following general procedure $\mathrm{E}(E)$-1-iodo-2-methyl-1-hexene ${ }^{12}(170 \mathrm{mg}, 0.76$ mmol), $t$-BuLi $(1.5 \mathrm{M}$ in pentane; $1.01 \mathrm{~mL}, 1.52 \mathrm{mmol})$ in THF $(2 \mathrm{~mL})$ then anhydrous $\mathrm{MgCl}_{2}(72 \mathrm{mg}, 0.76 \mathrm{mmol})$, a solution of LTMP $(1.08 \mathrm{mmol})$ in $\mathrm{Et}_{2} \mathrm{O}(8$ $\mathrm{mL}$ ) and then 1,2-epoxydodecane (100 $\mathrm{mg}, 0.54 \mathrm{mmol}$ ) gave after workup and column chromatography $\left(\mathrm{SiO}_{2}\right.$, petrol) $(E, E)$-diene $6 \mathbf{j}$ (101 mg, 70\% EE:ZE $=94: 6$ by GCMS analysis) as a colourless oil.

See above for data.

Table 4 The addition of excess organolithium to 1,2-epoxydodecane 5 without LTMP present ${ }^{a}$

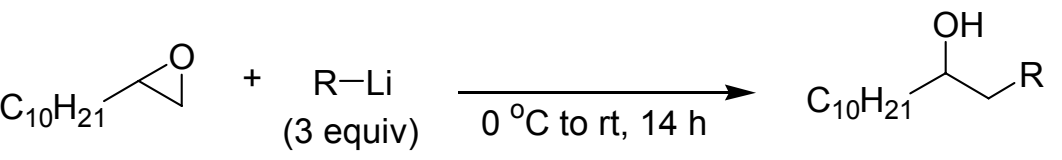

9

\begin{tabular}{|c|c|c|c|c|c|}
\hline Entry & $\mathrm{RLi}$ & Product & & Yield $(\%)^{b}$ & $E: Z^{c}$ \\
\hline 1 & PhLi & & $9 a$ & 69 & - \\
\hline 2 & & & $9 b$ & 72 & \\
\hline 3 & & & $9 c$ & 99 & $97: 3$ \\
\hline 4 & & & $9 d$ & 90 & $1: 99$ \\
\hline $5^{d}$ & & & $9 e$ & 75 & - \\
\hline 6 & & & $9 f$ & 70 & - \\
\hline
\end{tabular}

${ }^{a}$ Hexane as solvent unless otherwise indicated. ${ }^{b}$ isolated yield. ${ }^{c}$ Determined by GCMS. ${ }^{d}$ Reaction in $\mathrm{Et}_{2} \mathrm{O}$. 
General Procedure F: for alcohol formation using organolithiums, described for:

1-Phenyl-dodecan-2-ol 9a

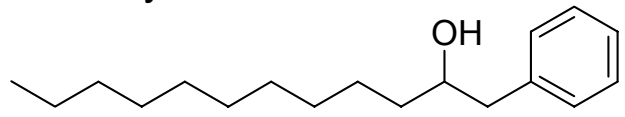

To a solution of 1,2-epoxydodecane $(100 \mathrm{mg}, 0.54 \mathrm{mmol})$ in hexane $(8 \mathrm{~mL})$ at $0{ }^{\circ} \mathrm{C}$ was added PhLi $\left(2.0 \mathrm{M}\right.$ in $\left.\mathrm{Bu}_{2} \mathrm{O} ; 0.82 \mathrm{~mL}, 1.63 \mathrm{mmol}\right)$ dropwise. The ice bath was removed and the reaction was left stirring for a $14 \mathrm{~h}$. After quenching with saturated brine solution $(5 \mathrm{~mL})$, the layers were separated. The aqueous layer was extracted with $\mathrm{Et}_{2} \mathrm{O}(2 \times 10 \mathrm{~mL})$, the combined organic layers were dried $\left(\mathrm{MgSO}_{4}\right)$ and solvent evaporated in vacuo. The residue was purified by column chromatography $\left(\mathrm{SiO}_{2}\right.$, $15 \% \mathrm{Et}_{2} \mathrm{O}$ in petrol) to give alcohol $9 \mathrm{a}(98 \mathrm{mg}, 69 \%)$ as a white solid; $\mathrm{R}_{\mathrm{f}} 0.16(10 \%$ $\mathrm{Et}_{2} \mathrm{O}$ in petrol), $\mathrm{mp}=40-41{ }^{\circ} \mathrm{C}$. IR $\left(\mathrm{CHCl}_{3}\right) / \mathrm{cm}^{-1} 3367 \mathrm{br}, 3027 \mathrm{~m}, 2919 \mathrm{~s}, 1496 \mathrm{w}$, 1466s, 1350w; ${ }^{1} \mathrm{H}$ NMR $(400 \mathrm{MHz}) \delta$ 7.35-7.31 (m, 2H), 7.27-7.22 (m, 3H), 3.85$3.79(\mathrm{~m}, 1 \mathrm{H}), 2.84(\mathrm{dd}, J=13.4,4.3,1 \mathrm{H}), 2.65(\mathrm{dd}, J=13.4,8.6,1 \mathrm{H}), 1.52-1.46(\mathrm{~m}$, $3 \mathrm{H}), 1.35-1.24(\mathrm{~m}, 16 \mathrm{H}), 0.90(\mathrm{t}, J=6.9,3 \mathrm{H}) ;{ }^{13} \mathrm{C}$ NMR $(100 \mathrm{MHz}) \delta 138.7,129.4$, $128.5,126.4,72.7,44.1,36.9,31.9,29.7,29.6,29.3,25.8,22.7,14.1 ; \mathrm{MS} \mathrm{m} / \mathrm{z}(\mathrm{Cl})$ $280\left(\mathrm{M}+\mathrm{NH}_{4}{ }^{+}, 100\right), 262$ (6), 244 (36), 169 (12), 117 (27), 104 (64), 92 (76), 91 (40); HRMS calcd for $\mathrm{C}_{18} \mathrm{H}_{34} \mathrm{NO}\left(\mathrm{M}+\mathrm{NH}_{4}{ }^{+}\right)$280.2640; found 280.2632.

Tetradec-1-en-4-ol ${ }^{17} \mathbf{9 b}$

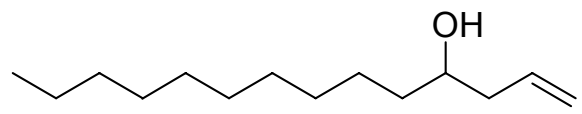

Following general procedure F, 1,2-epoxydodecane (100 mg, $0.54 \mathrm{mmol}$ ), vinyllithium $^{4}$ (1.5 $\mathrm{M}$ in $\left.\mathrm{Et}_{2} \mathrm{O} ; 1.09 \mathrm{~mL}, 1.63 \mathrm{mmol}\right)$ in hexane $(8 \mathrm{~mL})$ gave after workup and column chromatography $\left(\mathrm{SiO}_{2}, 15 \% \mathrm{Et}_{2} \mathrm{O}\right.$ in petrol) alcohol $\mathbf{9 b}$ (83 mg, $72 \%)$ as a white solid; $\mathrm{R}_{\mathrm{f}} 0.20\left(15 \% \mathrm{Et}_{2} \mathrm{O}\right.$ in petrol), $\mathrm{mp}=45-46{ }^{\circ} \mathrm{C}$. IR $\left(\mathrm{CHCl}_{3}\right) / \mathrm{cm}^{-1}$ 3341br, 2925s, 2854s, 1641w, 1466w, 994w, 912w; ${ }^{1} \mathrm{H}$ NMR (400 MHz) $\delta 5.89-5.78$ $(\mathrm{m}, 1 \mathrm{H}), 5.17-5.14(\mathrm{~m}, 1 \mathrm{H}), 5.13-5.11(\mathrm{~m}, 1 \mathrm{H}), 3.68-3.61(\mathrm{~m}, 1 \mathrm{H}), 2.34-2.27(\mathrm{~m}, 1 \mathrm{H})$, 2.18-2.10 (m, 1H), $1.63(\mathrm{br}, 1 \mathrm{H}), 1.48-1.22(\mathrm{~m}, 18 \mathrm{H}), 0.88(\mathrm{t}, J=7.1,3 \mathrm{H}) ;{ }^{13} \mathrm{C}$ NMR $(100 \mathrm{MHz}) \delta 134.9,118.0,70.7,41.9,36.8,31.9,29.7,29.6,29.3,25.7,22.7,14.1$; MS m/z (Cl) $230\left(\mathrm{M}+\mathrm{NH}_{4}, 100\right), 211$ (34), 169 (25), 97 (9); HRMS calcd for $\mathrm{C}_{14} \mathrm{H}_{32} \mathrm{NO}\left(\mathrm{M}+\mathrm{NH}_{4}{ }^{+}\right)$230.2484; found 230.2478.

${ }^{17}$ Chang, Y. H.; Uang, B. J; Wu, C.M.; Yu, T.H. Synthesis 1990, 1033-1034. 
(E)-Pentadec-2-en-5-ol 9c

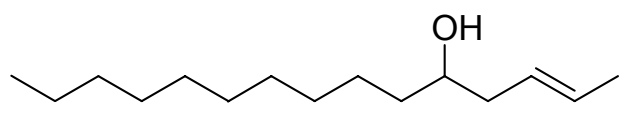

Following general procedure $\mathrm{F}, 1$,2-epoxydodecane $(100 \mathrm{mg}, 0.543 \mathrm{mmol}),(E)$ -

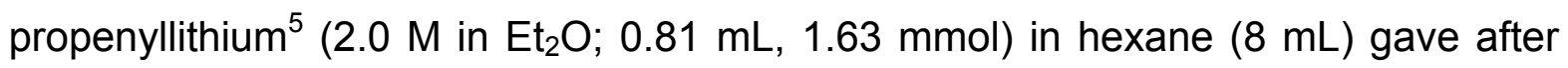
workup and column chromatography $\left(\mathrm{SiO}_{2}, 15 \% \mathrm{Et}_{2} \mathrm{O}\right.$ in petrol) alcohol 9c (122 mg, $99 \%, E: Z=97: 3$ by GCMS analysis, $\mathrm{t}_{R}(E)-9 \mathrm{c}=12.77 \mathrm{~min}, \mathrm{t}_{R}(Z)-9 \mathrm{c}=12.95 \mathrm{~min}$, initial temp. $=100{ }^{\circ} \mathrm{C}$, max. temp. $=280{ }^{\circ} \mathrm{C}$, rate $\left.=8{ }^{\circ} \mathrm{C} / \mathrm{min}\right)$ as a colourless oil; $R_{f}$ $0.16\left(10 \% \mathrm{Et}_{2} \mathrm{O}\right.$ in petrol). IR (neat)/cm ${ }^{-1} 3351 \mathrm{br}, 2925 \mathrm{~m}, 2855 \mathrm{~m}, 1466 \mathrm{w}, 967 \mathrm{w} ;{ }^{1} \mathrm{H}$ NMR $(400 \mathrm{MHz}) \delta 5.60-5.52(\mathrm{~m}, 1 \mathrm{H}), 5.48-5.40(\mathrm{~m}, 1 \mathrm{H}), 3.61-3.54(\mathrm{~m}, 1 \mathrm{H}), 2.27-$ $2.19(\mathrm{~m}, 1 \mathrm{H}), 2.09-2.01(\mathrm{~m}, 1 \mathrm{H}), 1.70(\mathrm{dd}, J=6.3,1.1,3 \mathrm{H}), 1.60(\mathrm{br}, 1 \mathrm{H}), 1.47-1.23$ $(\mathrm{m}, 18 \mathrm{H}), 0.88(\mathrm{t}, J=6.8,3 \mathrm{H}) ;{ }^{13} \mathrm{C}$ NMR $(100 \mathrm{MHz}) \delta 128.9,127.2,70.9,40.7,36.8$, 31.9, 29.7, 29.6, 29.3, 25.7, 22.7, 18.1, 14.1; MS m/z (Cl) $244\left(\mathrm{M}+\mathrm{NH}_{4}, 100\right), 242$ (63), 227 (35), 226 (37), 225 (63), 207 (20), 169 (41); HRMS calcd for $\mathrm{C}_{15} \mathrm{H}_{34} \mathrm{NO}$ $\left(\mathrm{M}+\mathrm{NH}_{4}{ }^{+}\right)$244.2640; found 244.2644.

\section{(Z)-Pentadec-2-en-5-ol 9d}

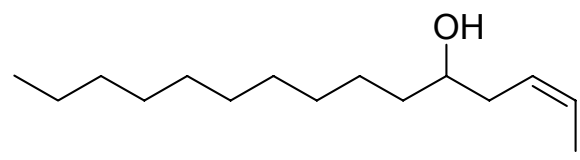

Following general procedure $F, 1,2$-epoxydodecane (100 $\mathrm{mg}, 0.54 \mathrm{mmol})$, (Z)-

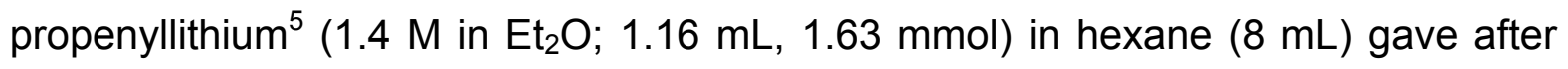
column chromatography $\left(\mathrm{SiO}_{2}, 15 \% \mathrm{Et}_{2} \mathrm{O}\right.$ in petrol $)$ alcohol $9 \mathrm{~d}(111 \mathrm{mg}, 90 \%, \mathrm{Z:E}=$ 99:1 by GCMS analysis, $\mathrm{t}_{R}(E)-9 \mathbf{d}=12.77 \mathrm{~min}, \mathrm{t}_{R}(Z)-9 \mathbf{d}=12.95 \mathrm{~min}$, initial temp. $=$ $100{ }^{\circ} \mathrm{C}$, max. temp. $=280{ }^{\circ} \mathrm{C}$, rate $\left.=8{ }^{\circ} \mathrm{C} / \mathrm{min}\right)$ as a colourless oil; $R_{\mathrm{f}} 0.16\left(10 \% \mathrm{Et}_{2} \mathrm{O}\right.$ in petrol). IR (neat) $/ \mathrm{cm}^{-1} 3349 \mathrm{br}, 3017 \mathrm{~m}, 2923 \mathrm{~s}, 2854 \mathrm{~s}, 1657 \mathrm{w}, 1467 \mathrm{~s}, 1077 \mathrm{~m}$, $1027 \mathrm{~m} ;{ }^{1} \mathrm{H}$ NMR $(400 \mathrm{MHz}) \delta 5.71-5.61(\mathrm{~m}, 1 \mathrm{H}), 5.49-5.40(\mathrm{~m}, 1 \mathrm{H}), 3.64$ (quint., $J=$ 6.1, 1H), $2.23(\mathrm{t}, J=6.1,2 \mathrm{H}), 1.65(\mathrm{~d}, J=6.8,3 \mathrm{H}), 1.57(\mathrm{br}, 1 \mathrm{H}), 1.52-1.20(\mathrm{~m}$, $18 \mathrm{H}), 0.89(\mathrm{t}, J=6.8,3 \mathrm{H}) ;{ }^{13} \mathrm{C} \mathrm{NMR}(100 \mathrm{MHz}) \delta 127.3,126.2,71.5,36.9,35.0$, 31.9, 29.7, 29.6, 29.3, 25.8, 22.7, 14.1, 13.0; MS m/z (Cl) $244\left(\mathrm{M}+\mathrm{NH}_{4}, 100\right), 242$ (81), 227 (30), 226 (40), 225 (69), 208 (25), 169 (19); HRMS calcd for $\mathrm{C}_{15} \mathrm{H}_{34} \mathrm{NO}$ $\left(\mathrm{M}+\mathrm{NH}_{4}{ }^{+}\right)$244.2640; found 244.2639. 
1-Trimethylsilanyl-tridecan-3-ol 9e

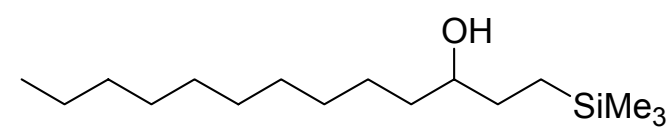

Following general procedure F, 1,2-epoxydodecane (100 $\mathrm{mg}, 0.54 \mathrm{mmol}$ ) and (trimethylsilylmethyl)lithium (1.0 M in pentane, $1.63 \mathrm{~mL}, 1.63 \mathrm{mmol}$ ) in hexane (8 $\mathrm{mL})$ gave after column chromatography $\left(\mathrm{SiO}_{2}, 10 \% \mathrm{Et}_{2} \mathrm{O}\right.$ in petrol) alcohol $9 \mathrm{e}$ (111 $\mathrm{mg}, 75 \%)$ as a colourless oil; $\mathrm{R}_{\mathrm{f}} 0.20$ (10\% $\mathrm{Et}_{2} \mathrm{O}$ in petrol). IR (neat)/ $/ \mathrm{cm}^{-1} 3346 \mathrm{br}$, 2925s, 2854s, 1466m, 1378m, 1248s, 1179m, 1127m, 1076m, 1007m; ${ }^{1} \mathrm{H}$ NMR $(400 \mathrm{MHz}) \delta 3.50-3.44(\mathrm{~m}, 1 \mathrm{H}), 1.74(\mathrm{br}, 1 \mathrm{H}), 1.48-1.20(\mathrm{~m}, 20 \mathrm{H}), 0.86(\mathrm{t}, \mathrm{J}=6.8$, $3 \mathrm{H}$ ), 0.59 (ddd, $J=13.9,13.9,4.6,1 \mathrm{H}) 0.40$ (ddd, $J=13.9,12.9,5.1,1 \mathrm{H}$ ), -0.02 (s, 9H); ${ }^{13} \mathrm{C}$ NMR $(100 \mathrm{MHz}) \delta 74.1,36.7,31.9,31.5,29.8,29.6,29.3,25.7$, 22.7, 14.1, 12.0, -1.8; MS m/z (Cl) $290\left(\mathrm{M}+\mathrm{NH}_{4}{ }^{+}, 100\right), 288$ (18), 272 (25), 92 (26), 91 (34), 90 (83); HRMS calcd for $\mathrm{C}_{16} \mathrm{H}_{40} \mathrm{NOSi}\left(\mathrm{M}+\mathrm{NH}_{4}{ }^{+}\right)$290.2874; found 290.2877.

\section{6-Trimethylsilanyl-octadecan-8-ol 9f}

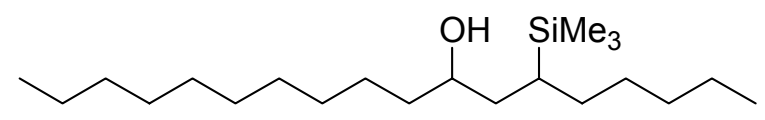

Following general procedure F, 1,2-epoxydodecane (100 mg, $0.54 \mathrm{mmol}$ ) and 1(trimethylsilyl)hexyllithium (1.1 M in THF, $1.79 \mathrm{~mL}, 1.63 \mathrm{mmol})$ in hexane $(8 \mathrm{~mL})$ gave after column chromatography $\left(\mathrm{SiO}_{2}, 10 \% \mathrm{Et}_{2} \mathrm{O}\right.$ in petrol) alcohol 9 f $(130 \mathrm{mg}$, $70 \% 50: 50$ diastereoisomers by GC analysis, $\mathrm{t}_{R}=11.23 \mathrm{~min}, \mathrm{t}_{R}=11.28 \mathrm{~min}$, initial temp. $=80{ }^{\circ} \mathrm{C}$, max. temp. $=280{ }^{\circ} \mathrm{C}$, rate $=20{ }^{\circ} \mathrm{C} / \mathrm{min}$ ) as a colourless wax; $\mathrm{R}_{\mathrm{f}} 0.24$ (10\% $\mathrm{Et}_{2} \mathrm{O}$ in petrol). IR (neat)/cm $\mathrm{cm}^{-1} 3360 \mathrm{br}, 2925 \mathrm{~s}, 2855 \mathrm{~s}, 1730 \mathrm{w}, 1467 \mathrm{~m}, 1378 \mathrm{w}$, 1248s, 834m; ${ }^{1} \mathrm{H}$ NMR (400 MHz) $\delta 3.70-3.60$ (m, 1H), 1.60 (br, 1H), 1.58-1.20 (m, $27 \mathrm{H}), 0.88(\mathrm{t}, J=6.6,6 \mathrm{H}), 0.81-0.75(\mathrm{~m}, 1 \mathrm{H}), 0.69-0.62(\mathrm{~m}, 1 \mathrm{H}),-0.01(\mathrm{~s}, 9 \mathrm{H}) ;{ }^{13} \mathrm{C}$ NMR $(100 \mathrm{MHz}) \delta 71.5,70.0,38.2,37.7,37.3,32.5,32.3,31.9,29.8,29.7,29.7$, 29.6, 29.3, 29.0, 28.8, 25.8, 25.6, 22.7, 22.6, 22.6, 22.2, 21.9, 14.1, -2.2, -2.3; MS m/z (Cl) $360\left(\mathrm{M}+\mathrm{H}^{+}, 52\right), 325$ (5), 90 (100); HRMS: m/z calcd for $\mathrm{C}_{21} \mathrm{H}_{50} \mathrm{SiNO}$ $\left(\mathrm{M}+\mathrm{NH}_{4}^{+}\right)$360.3656; found 360.3651 . 
${ }^{1} \mathrm{H}$ and ${ }^{13} \mathrm{C}$ NMR for $\mathbf{6 a}$

禹事
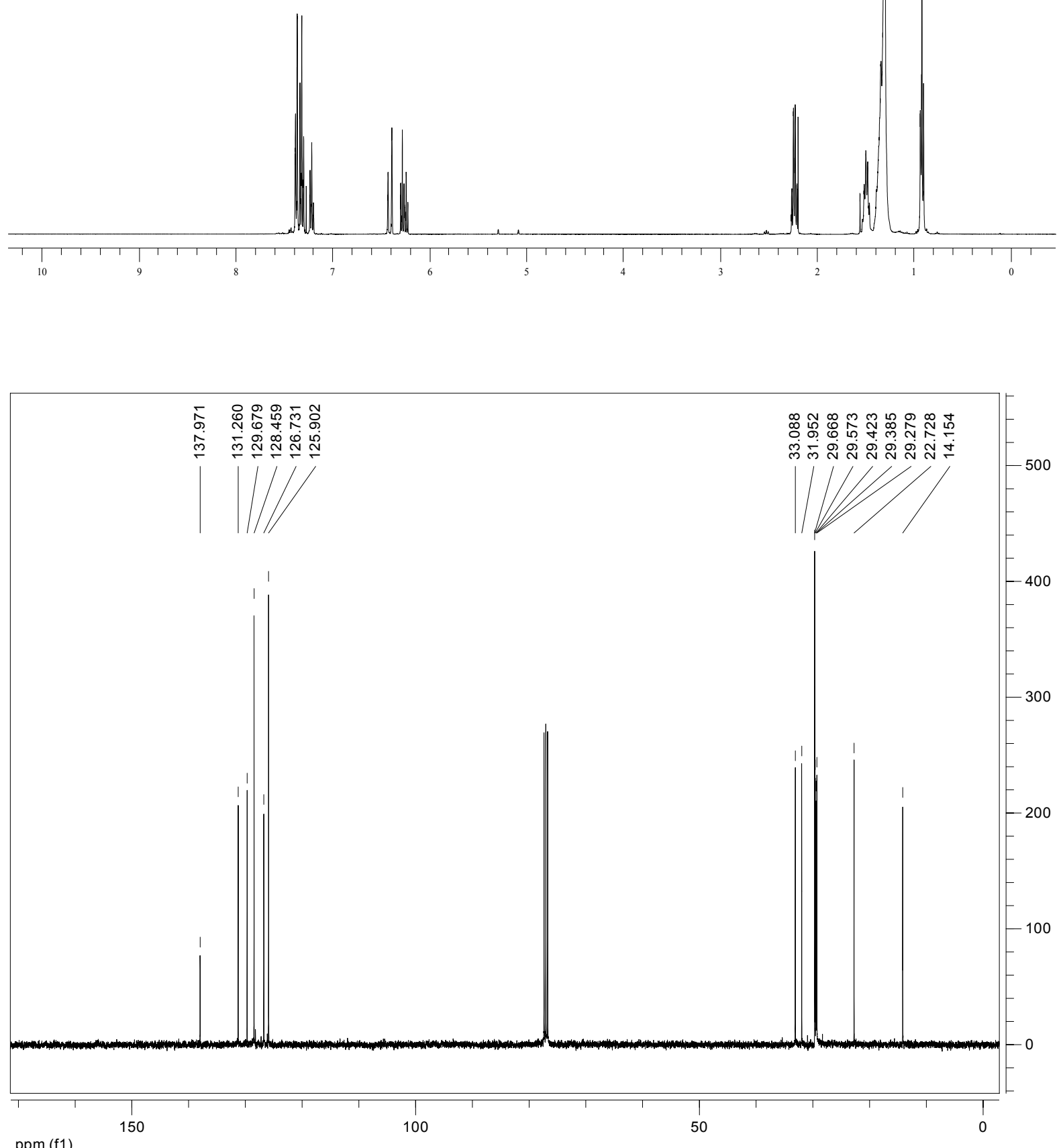
${ }^{1} \mathrm{H}$ and ${ }^{13} \mathrm{C}$ NMR for $\mathbf{6 b}$

$\overbrace{}^{0}$

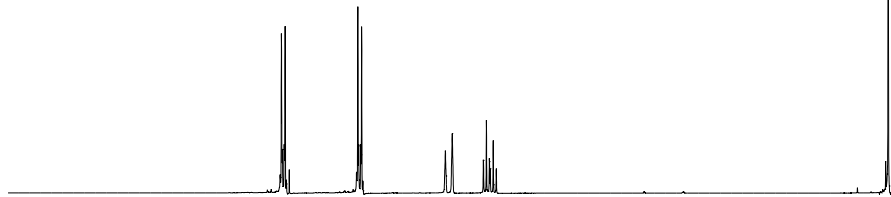

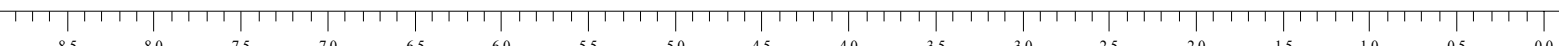

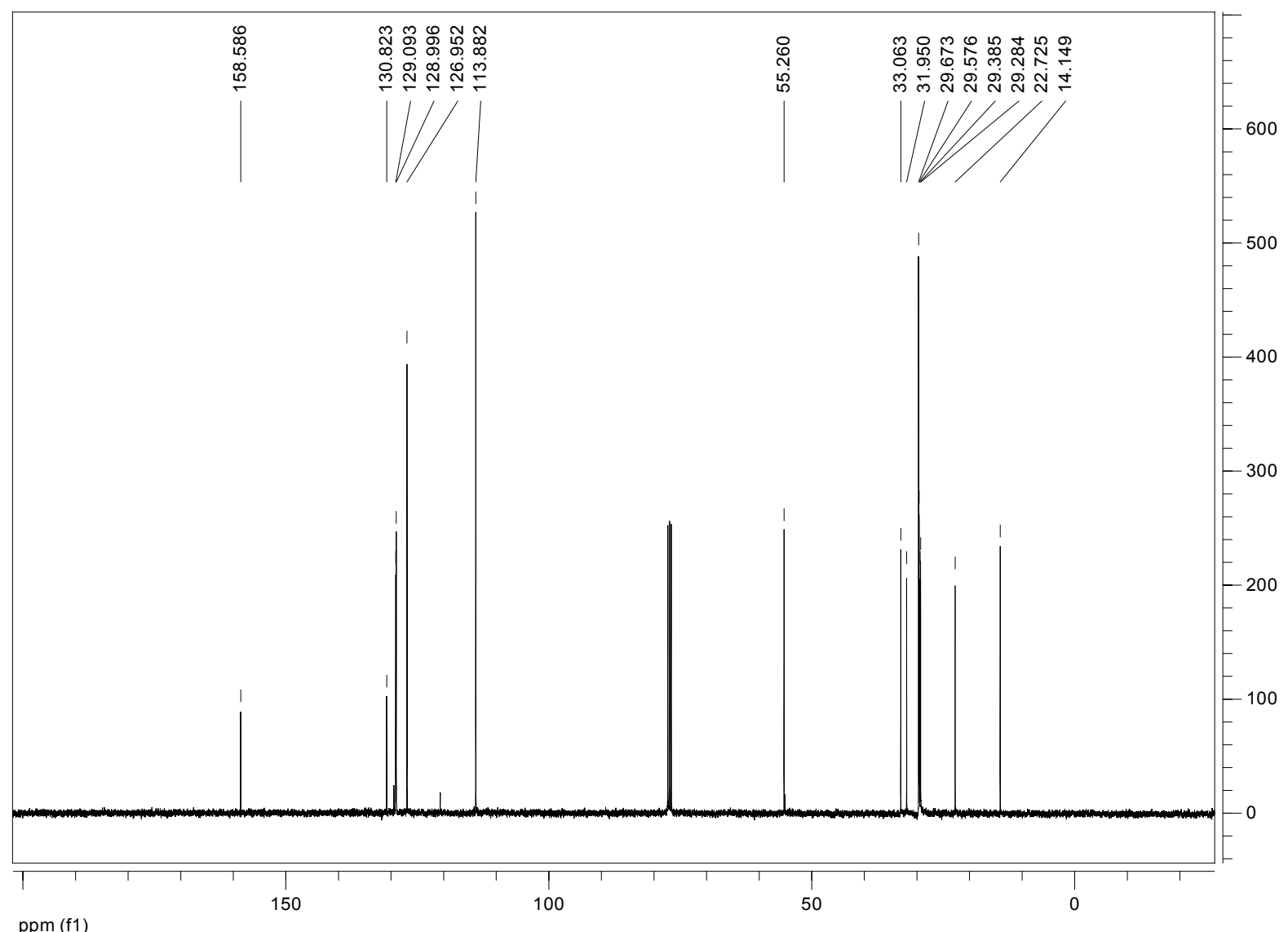




\section{${ }^{1} \mathrm{H}$ and ${ }^{13} \mathrm{C}$ NMR for $\mathbf{6 c}$}<smiles>C=CC=CCCCCCCCCCC</smiles>

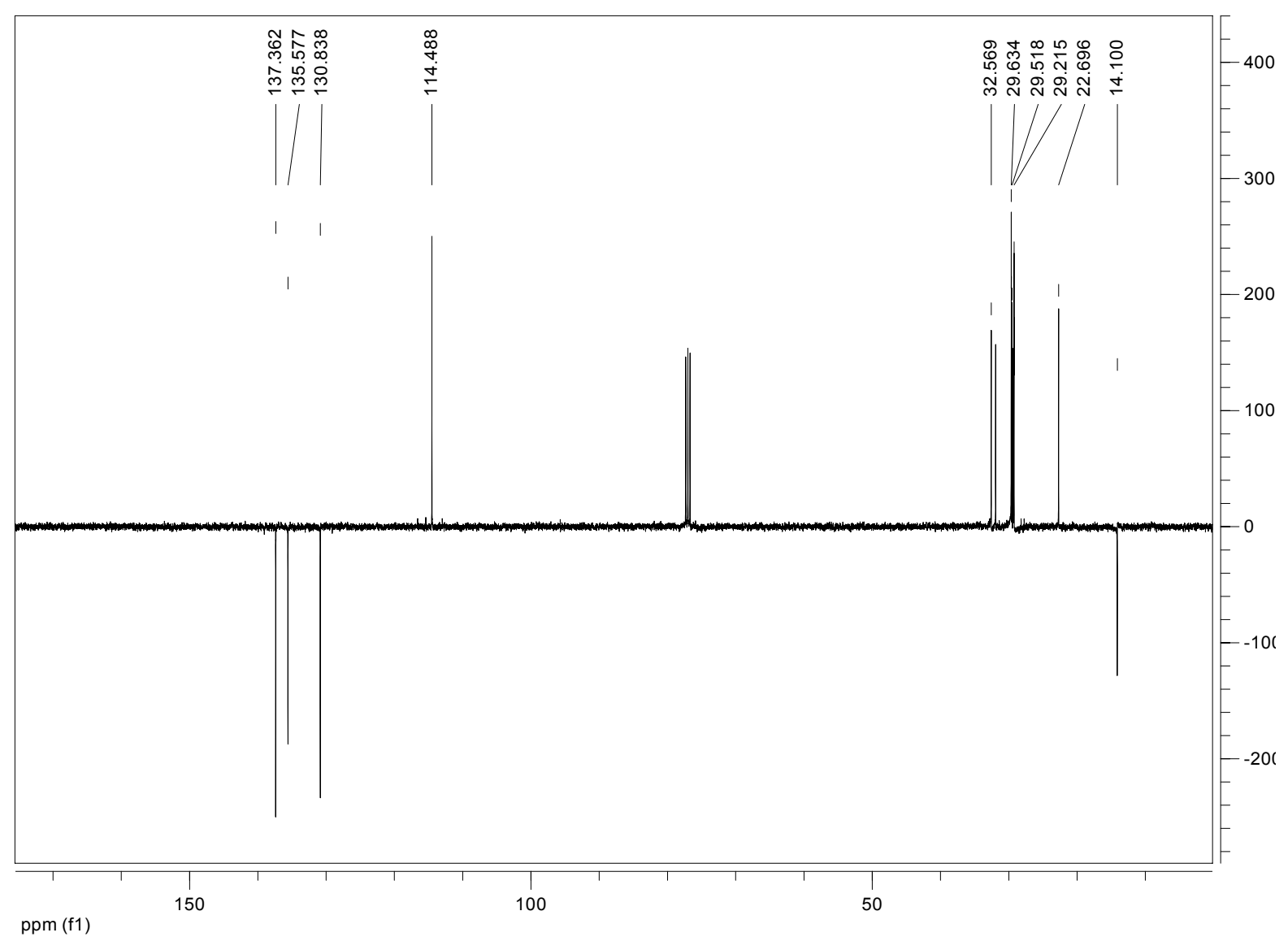


${ }^{1} \mathrm{H}$ and ${ }^{13} \mathrm{C}$ NMR for $\mathbf{6 d}$
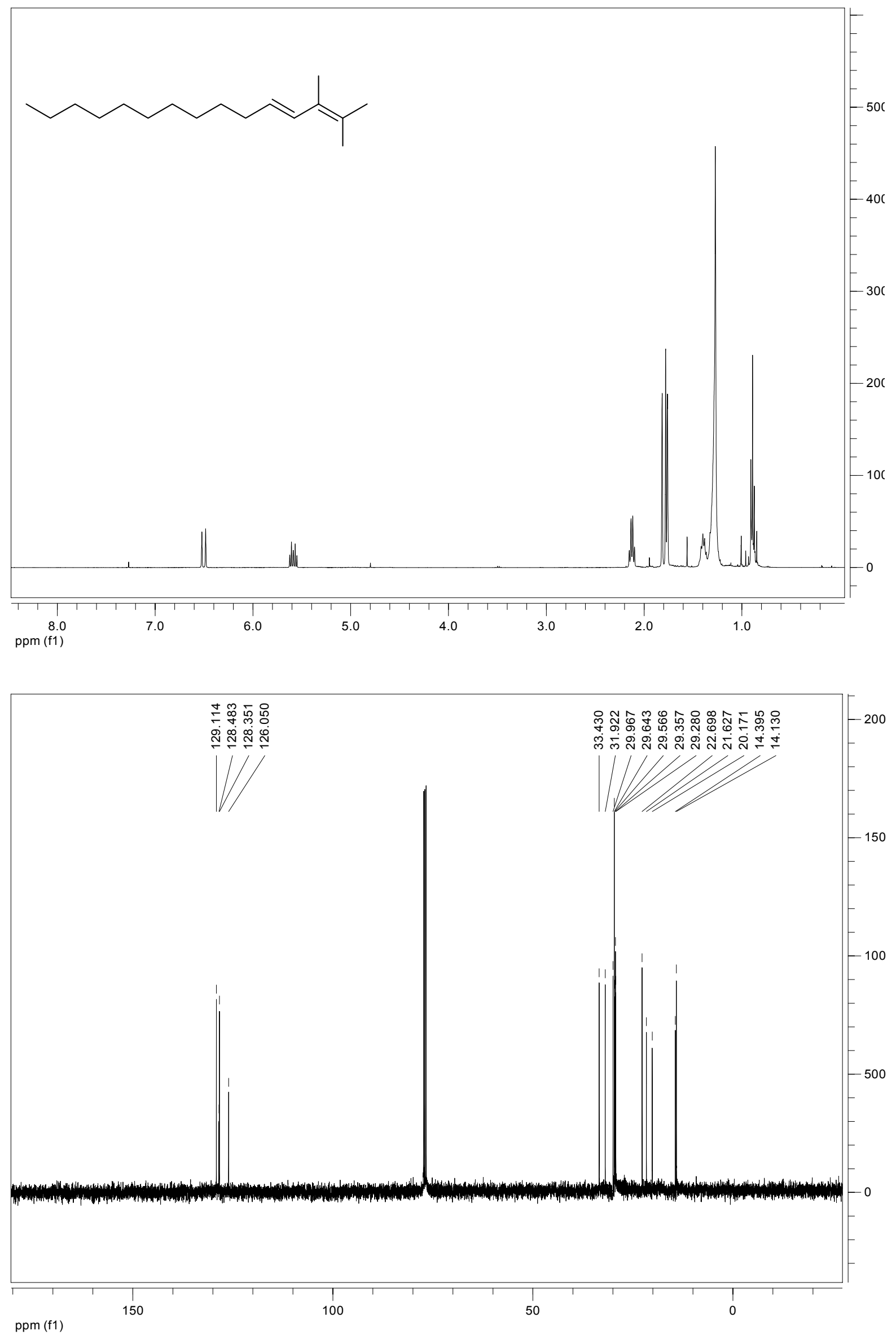


\section{S 22}

${ }^{1} \mathrm{H}$ and ${ }^{13} \mathrm{C}$ NMR for $6 \mathrm{e}$

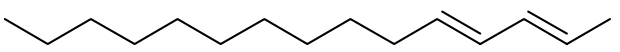
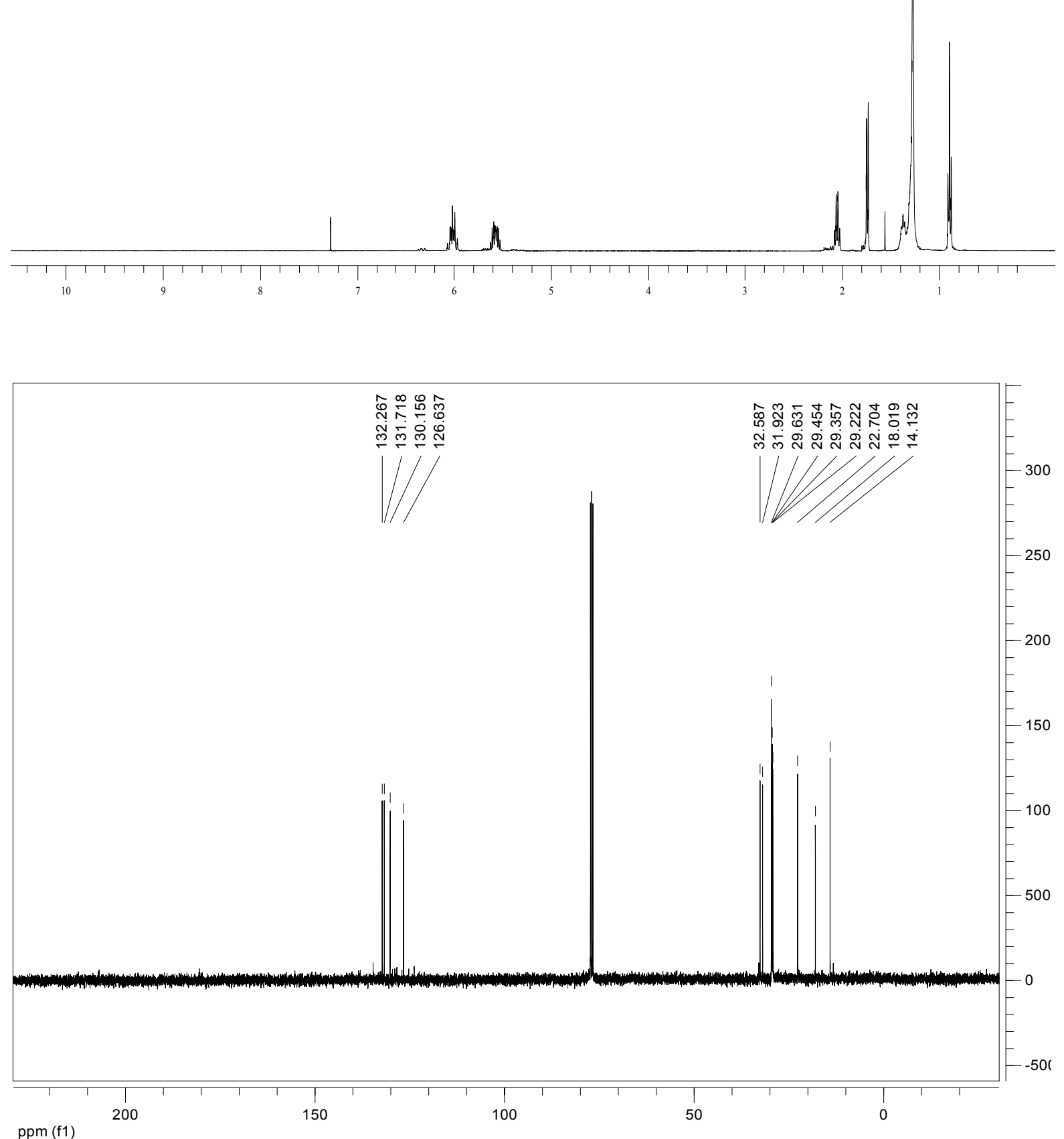
${ }^{1} \mathrm{H}$ and ${ }^{13} \mathrm{C}$ NMR for $6 \mathbf{f}$

疗
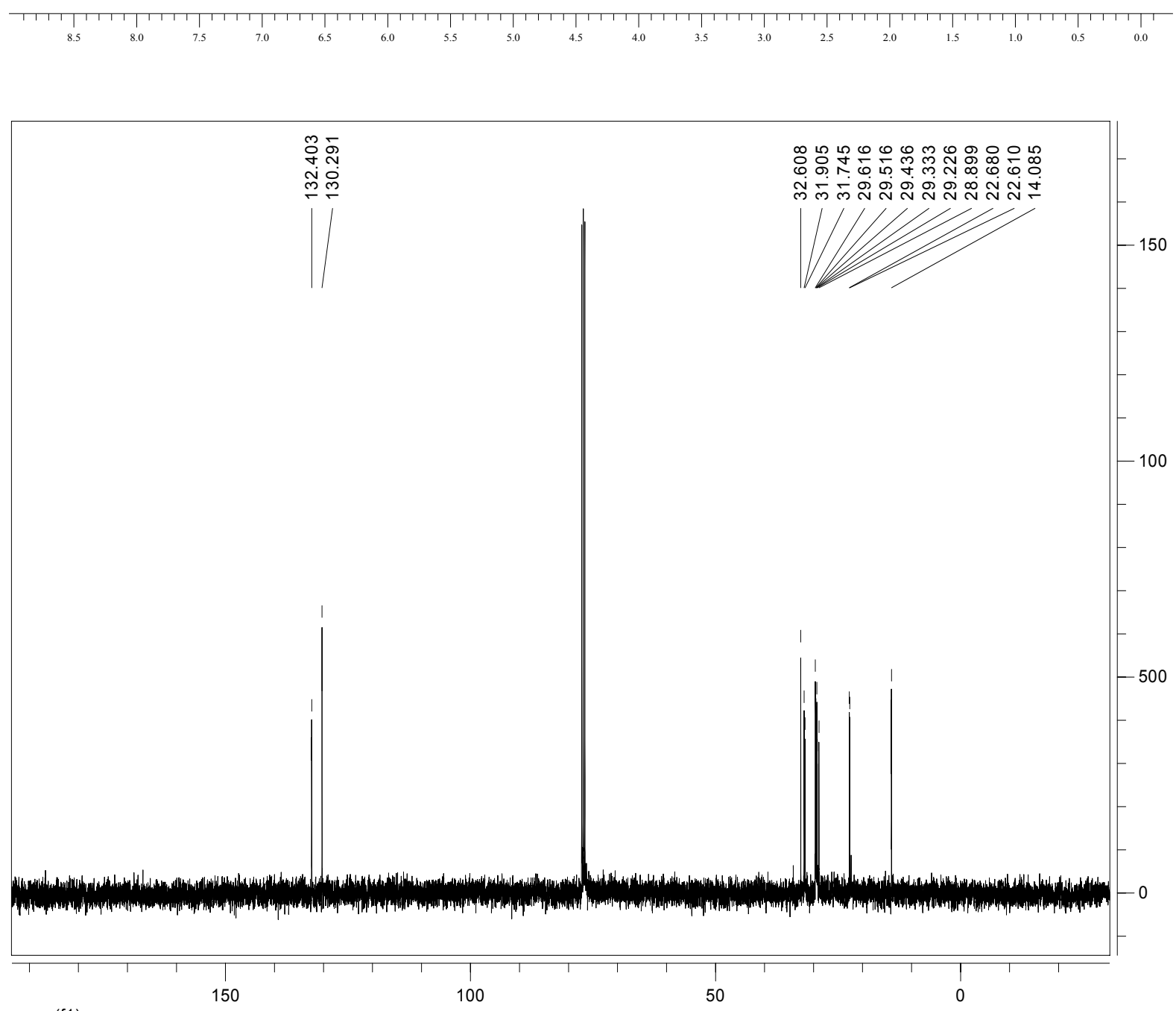

ppm (f1) 
${ }^{1} \mathrm{H}$ and ${ }^{13} \mathrm{C}$ NMR for $\mathbf{6 g}$

禹事

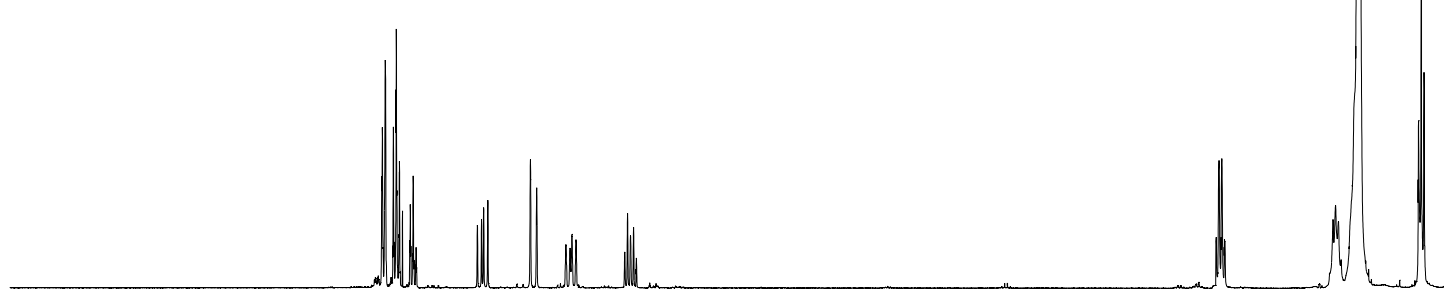

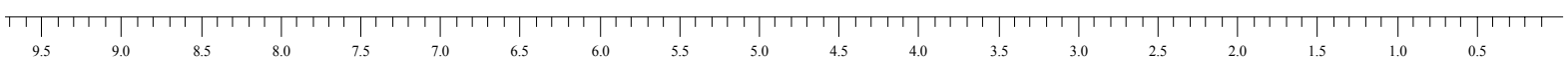

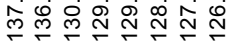

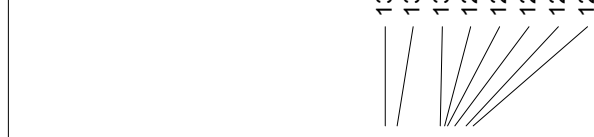

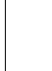
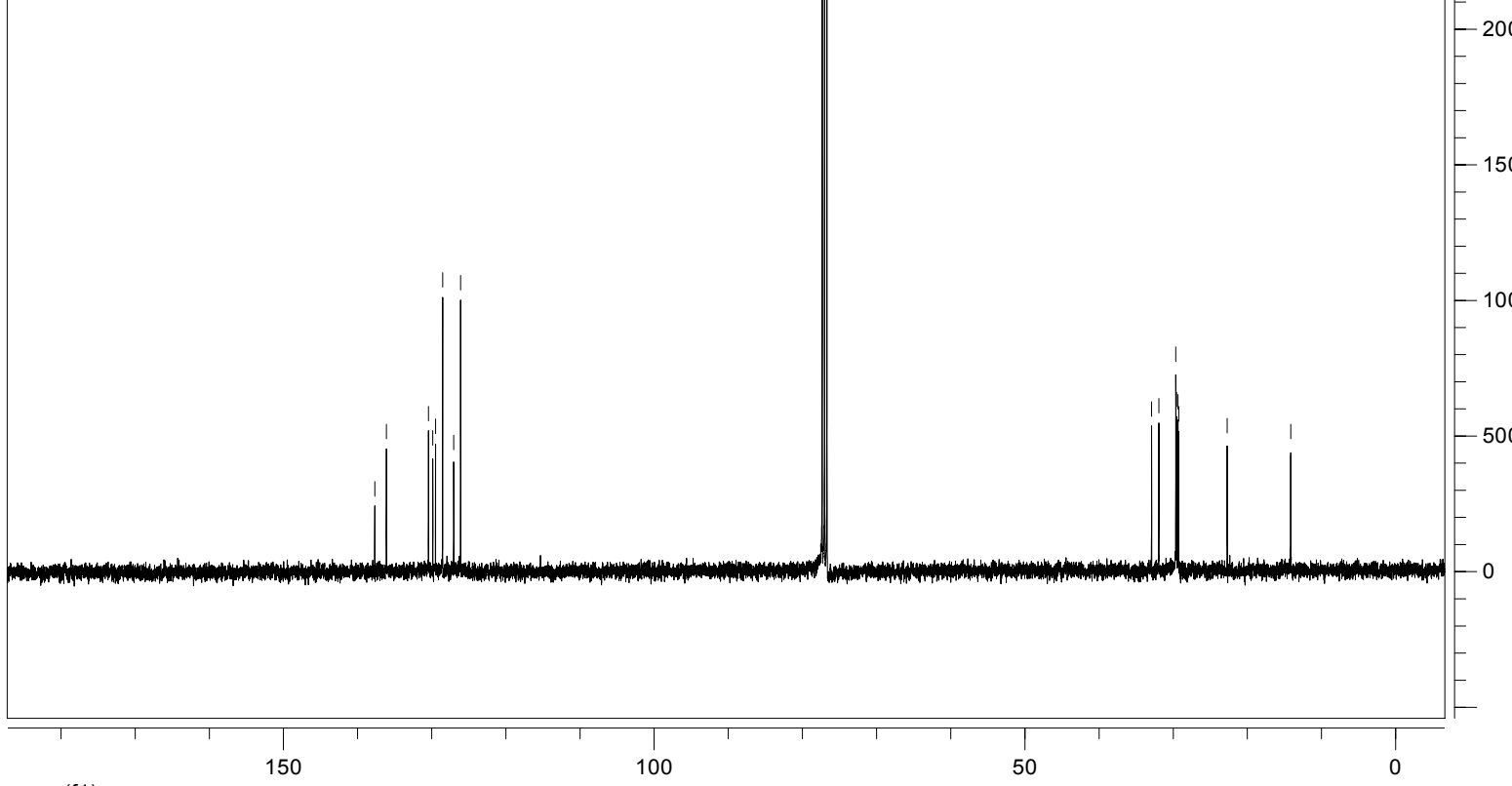

ppm (f1)

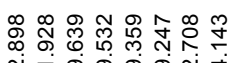

लिं

$-300$

$1 / 1$ 


\section{S 25}

${ }^{1} \mathrm{H}$ and ${ }^{13} \mathrm{C}$ NMR for $6 \mathrm{~h}$

N

茴 容

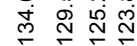

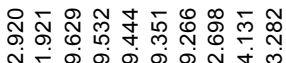

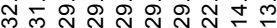
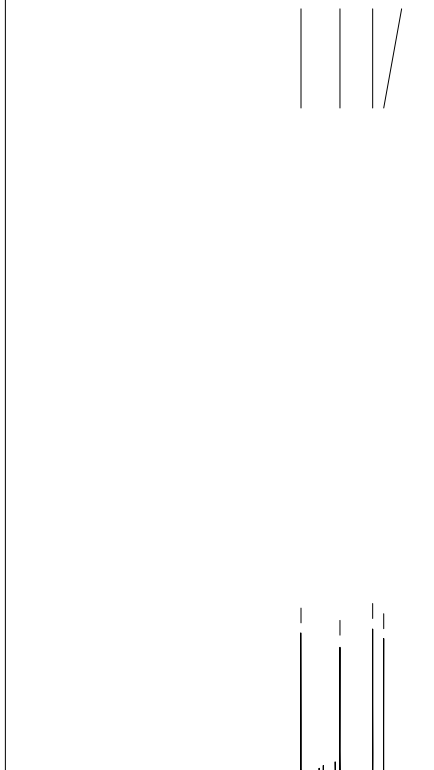

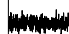

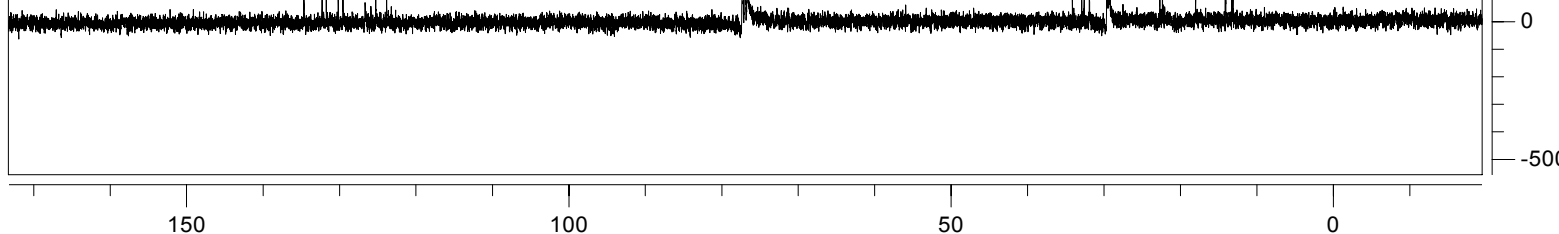

ppm (f1) 
${ }^{1} \mathrm{H}$ and ${ }^{13} \mathrm{C}$ NMR for $6 \mathbf{i}$

NON
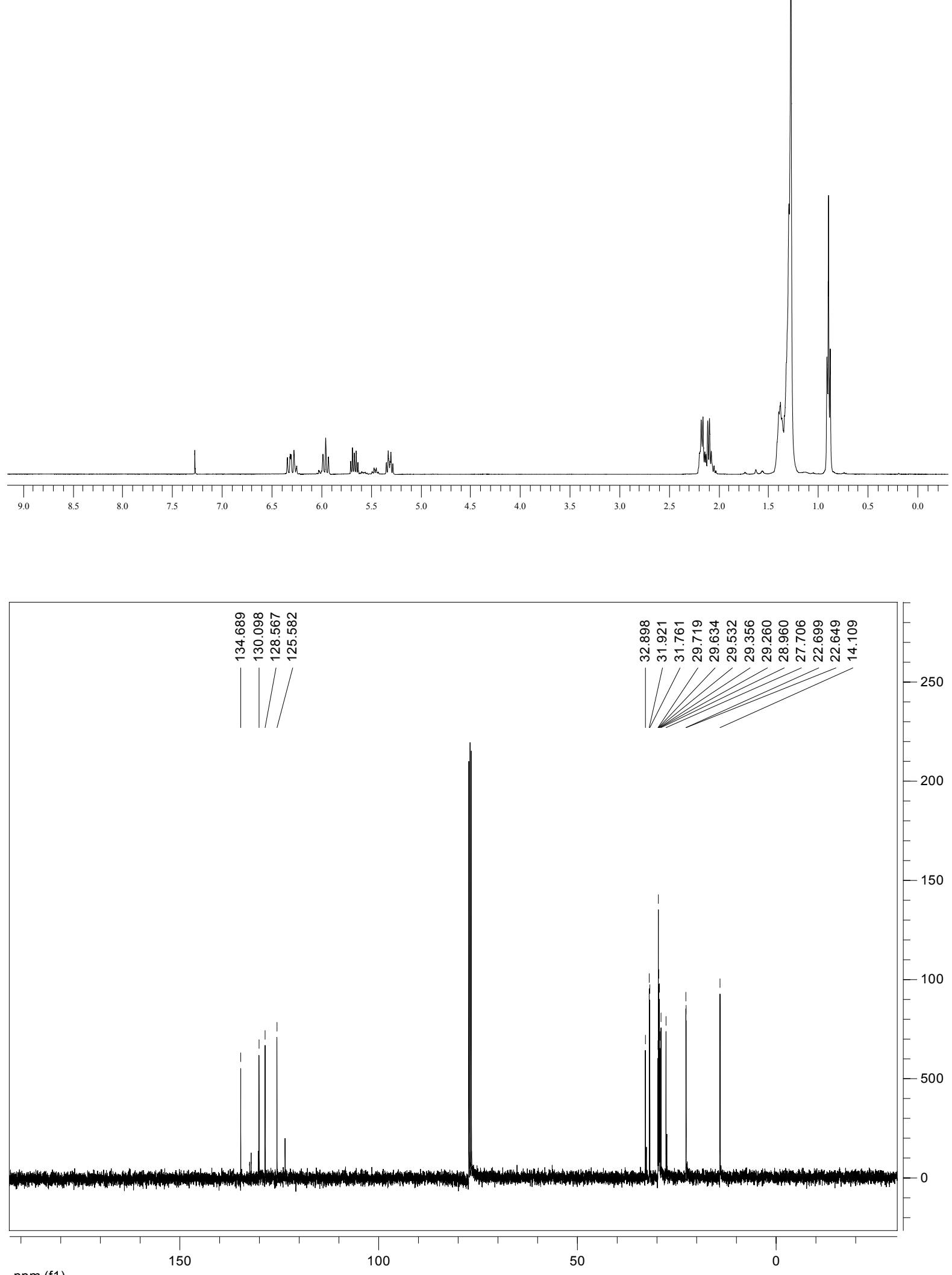

ppm (f1) 
${ }^{1} \mathrm{H}$ and ${ }^{13} \mathrm{C}$ NMR for $\mathbf{6 j}$

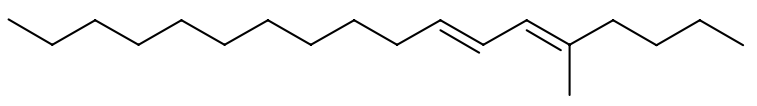
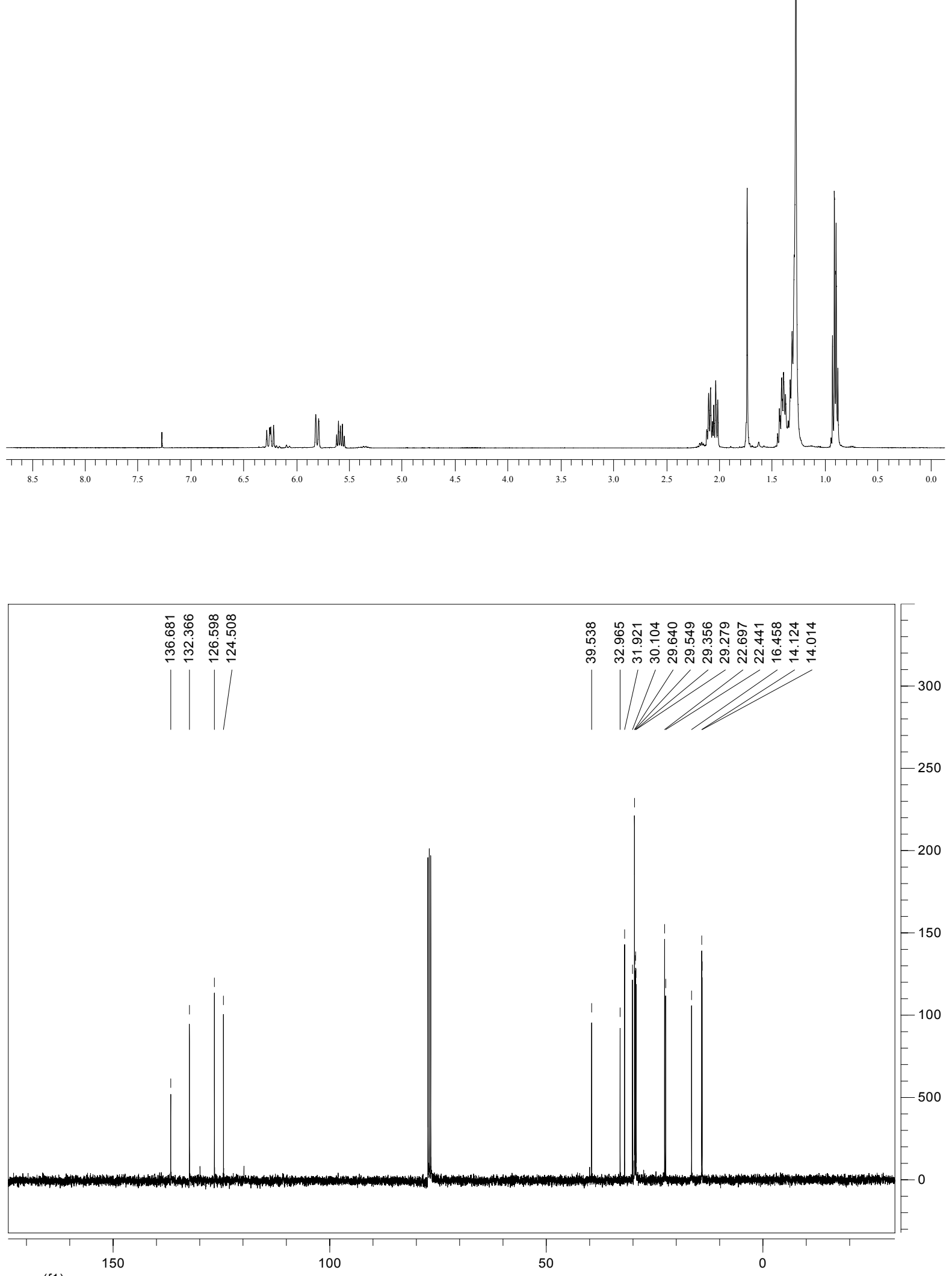

ppm (f1) 
${ }^{1} \mathrm{H}$ and ${ }^{13} \mathrm{C}$ NMR for $7 \mathrm{a}$

$\mathrm{SiMe}_{3}$
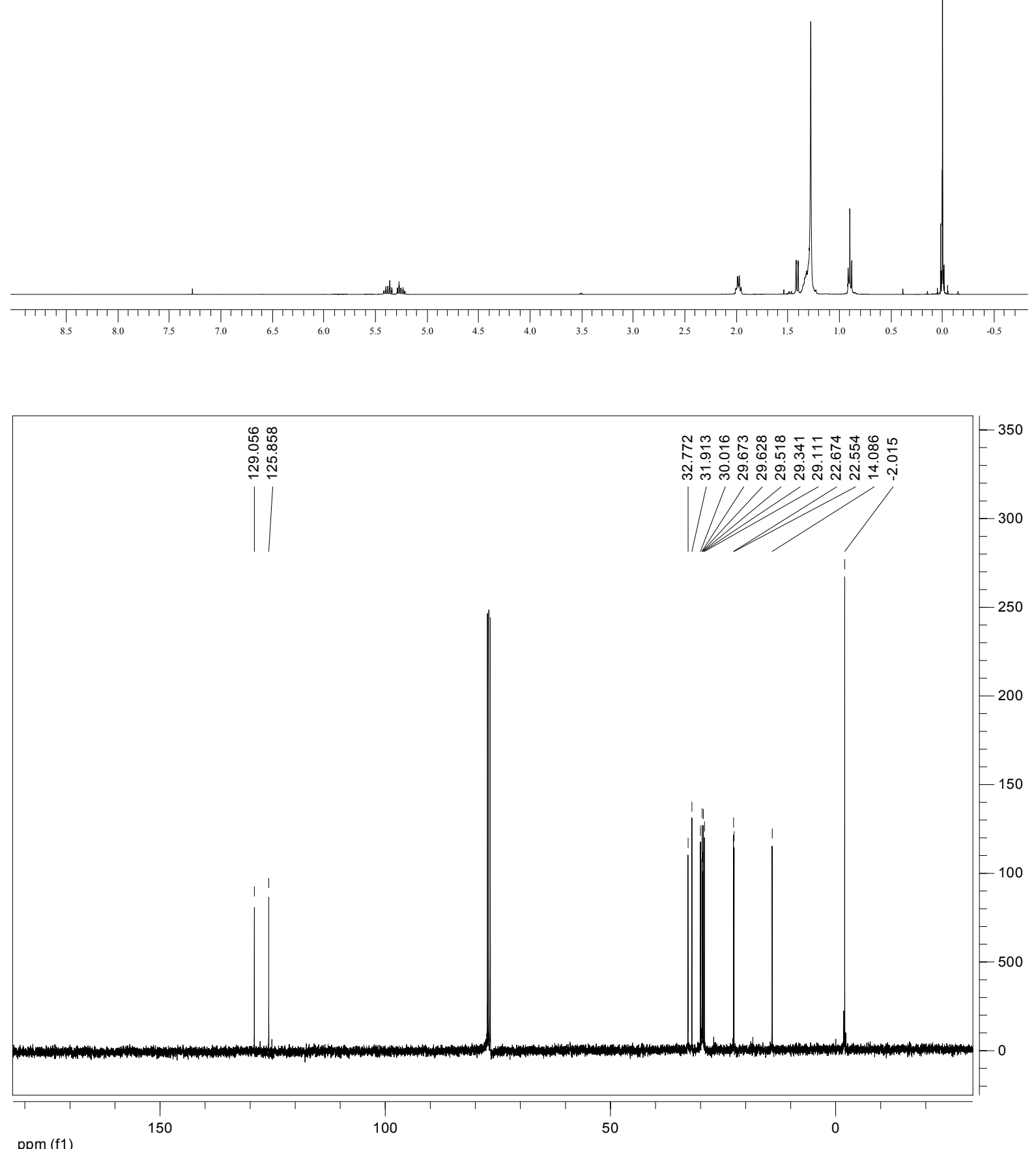

ppm (f1) 


\section{S 29}

${ }^{1} \mathrm{H}$ and ${ }^{13} \mathrm{C}$ NMR for $\mathbf{7 b}$

$\sim_{\mathrm{SiMe}_{3}}$

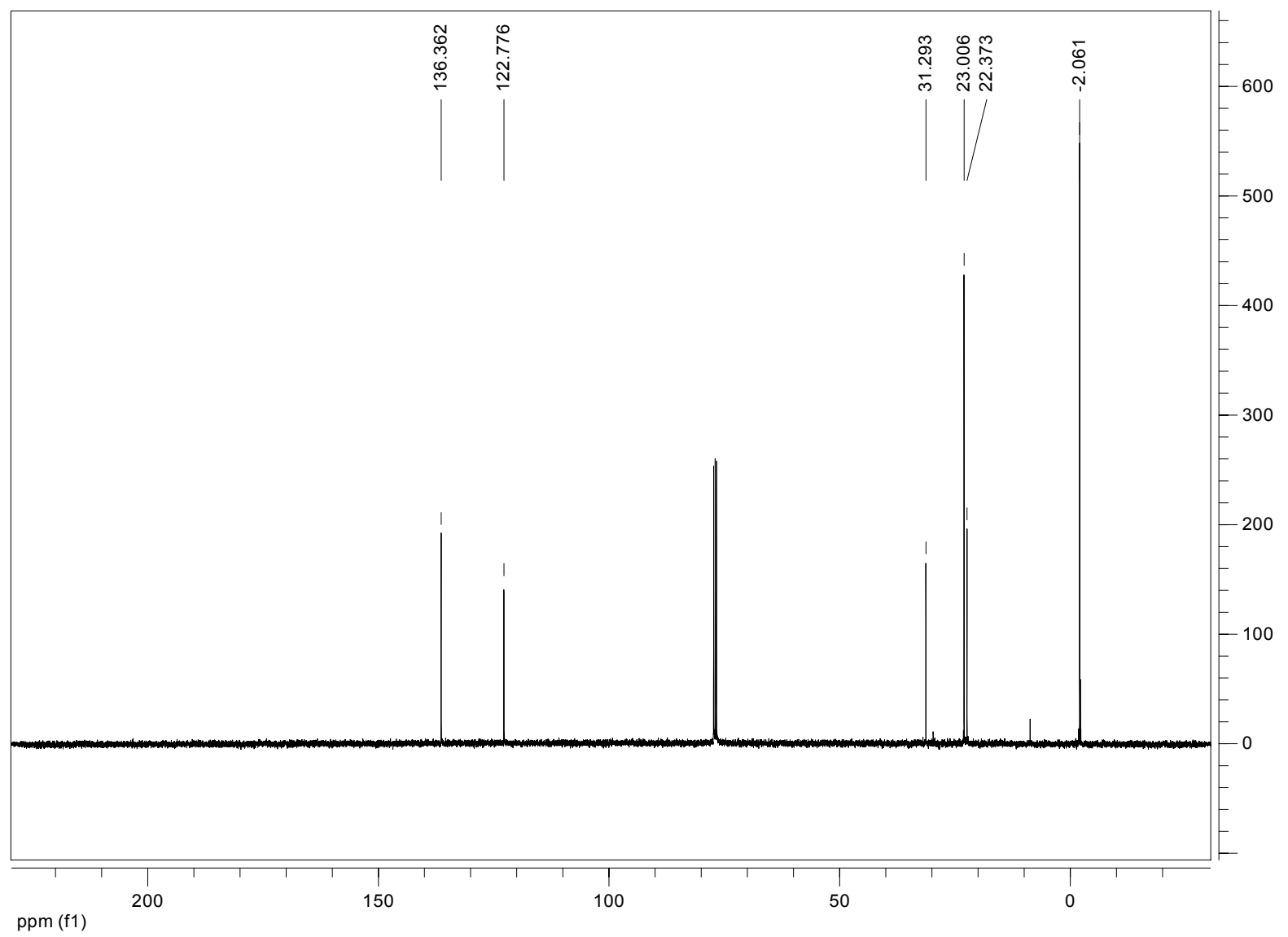


${ }^{1} \mathrm{H}$ and ${ }^{13} \mathrm{C}$ NMR for $7 \mathrm{c}$

$\curvearrowright \mathrm{SiMe}_{3}$

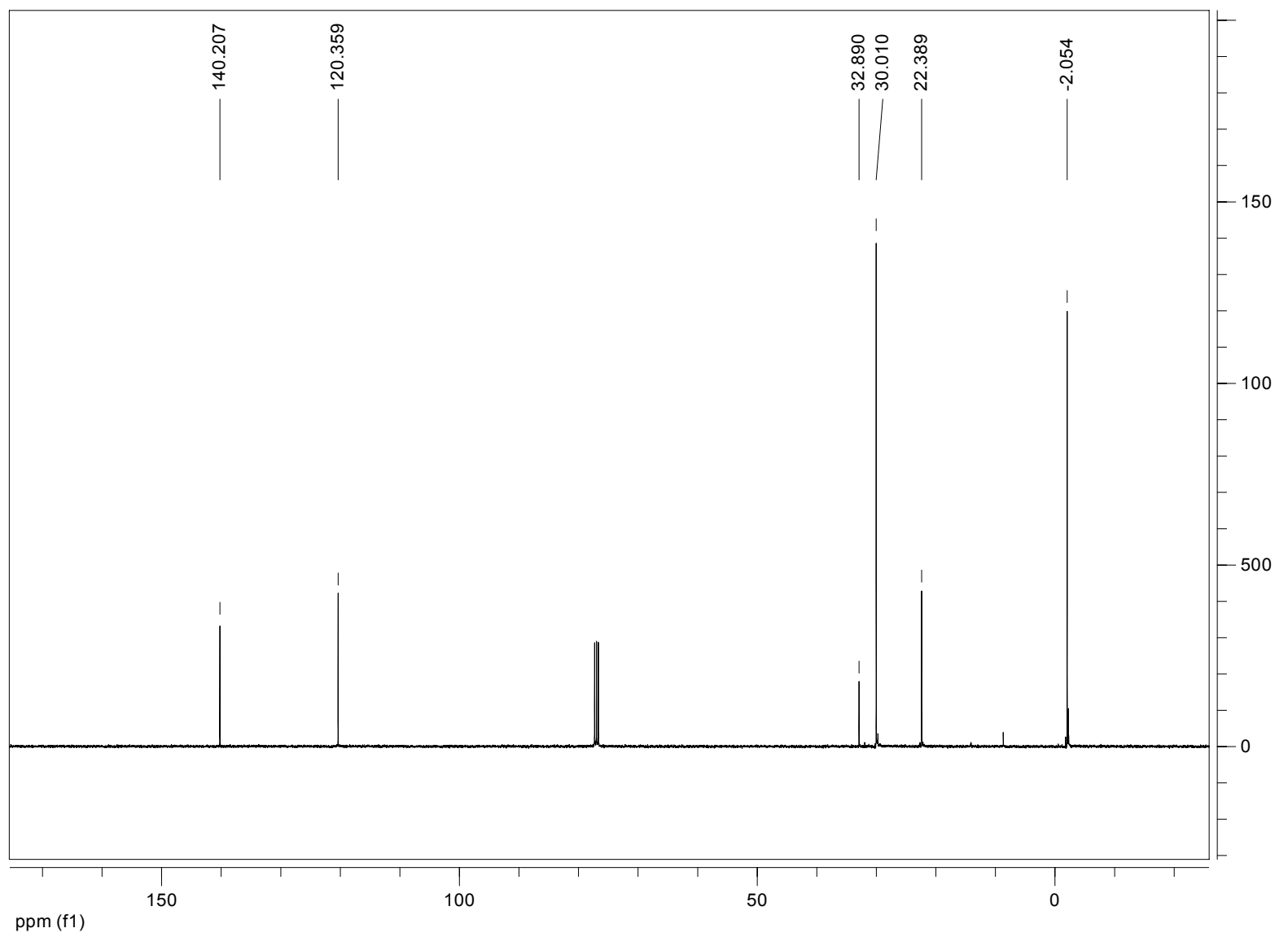




\section{S 31}

${ }^{1} \mathrm{H}$ and ${ }^{13} \mathrm{C}$ NMR for $7 \mathrm{~d}$

(N)
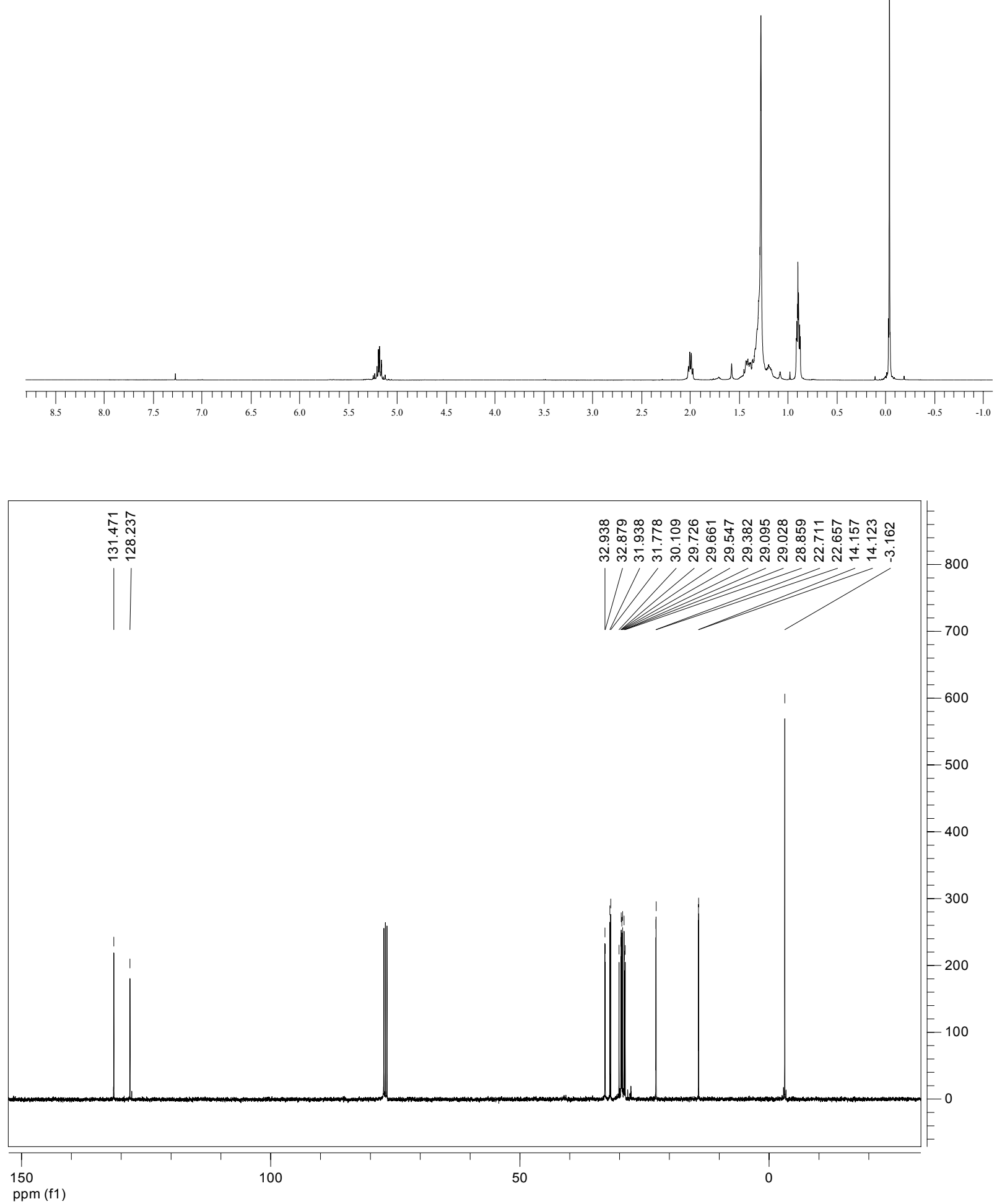
${ }^{1} \mathrm{H}$ and ${ }^{13} \mathrm{C}$ NMR for $7 e$

$\overbrace{}^{\mathrm{SiMe}_{3}}$
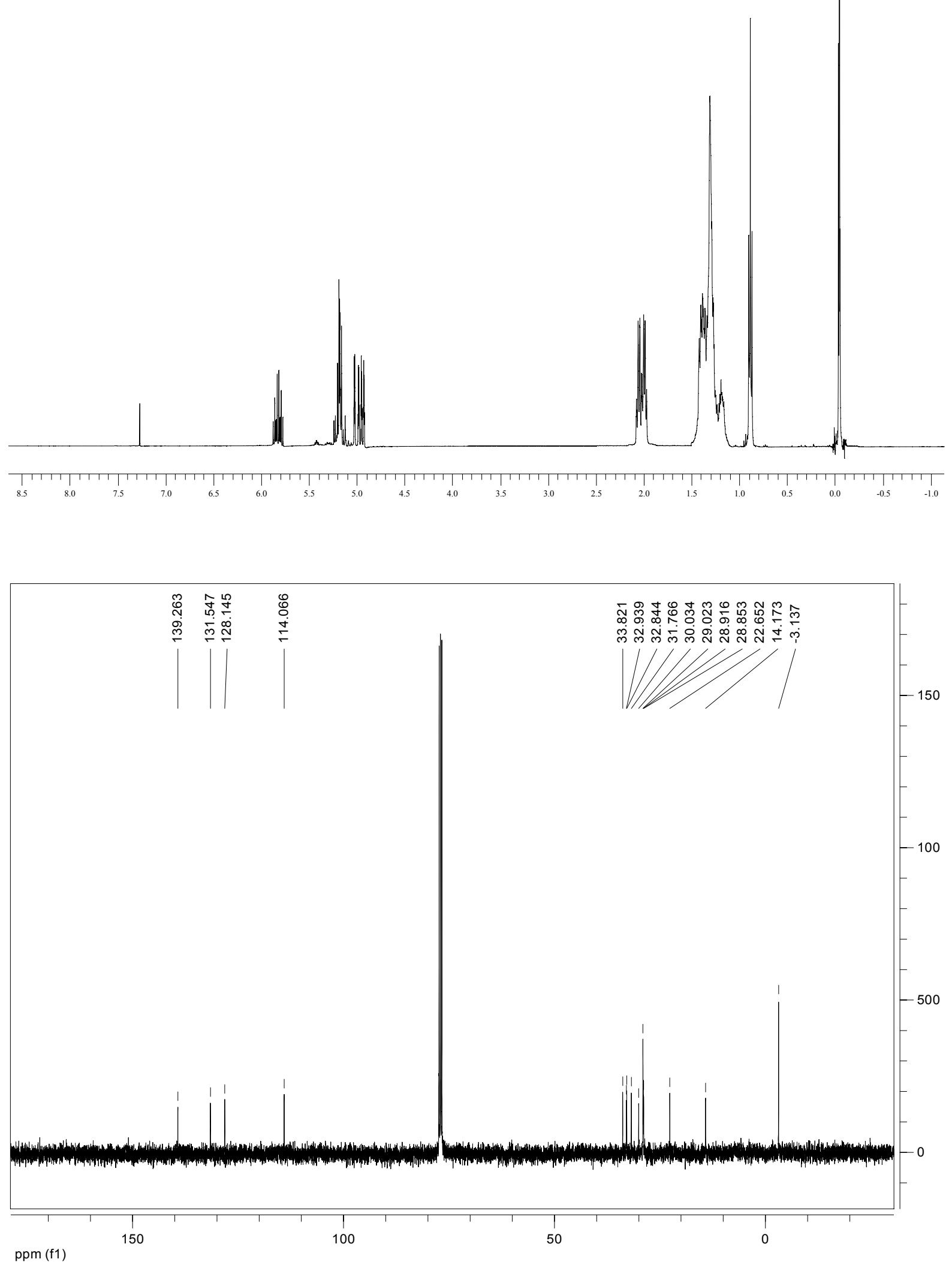
${ }^{1} \mathrm{H}$ and ${ }^{13} \mathrm{C}$ NMR for $\mathbf{7 f}$<smiles>CCCCCC(C)/C=C/CCc1ccccc1</smiles>
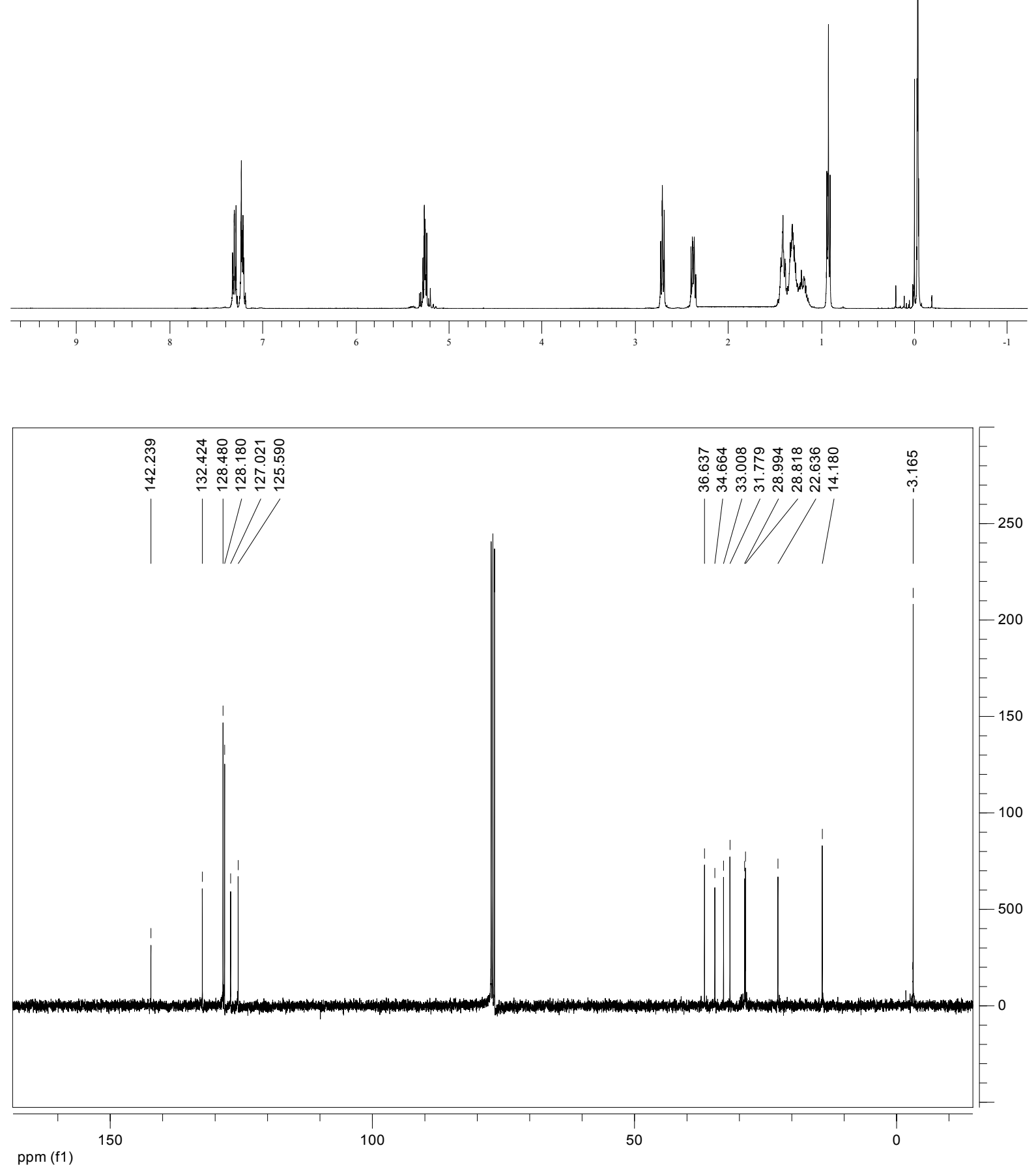


\section{S 34}

${ }^{1} \mathrm{H}$ and ${ }^{13} \mathrm{C}$ NMR for $\mathbf{7 g}$

SBSO
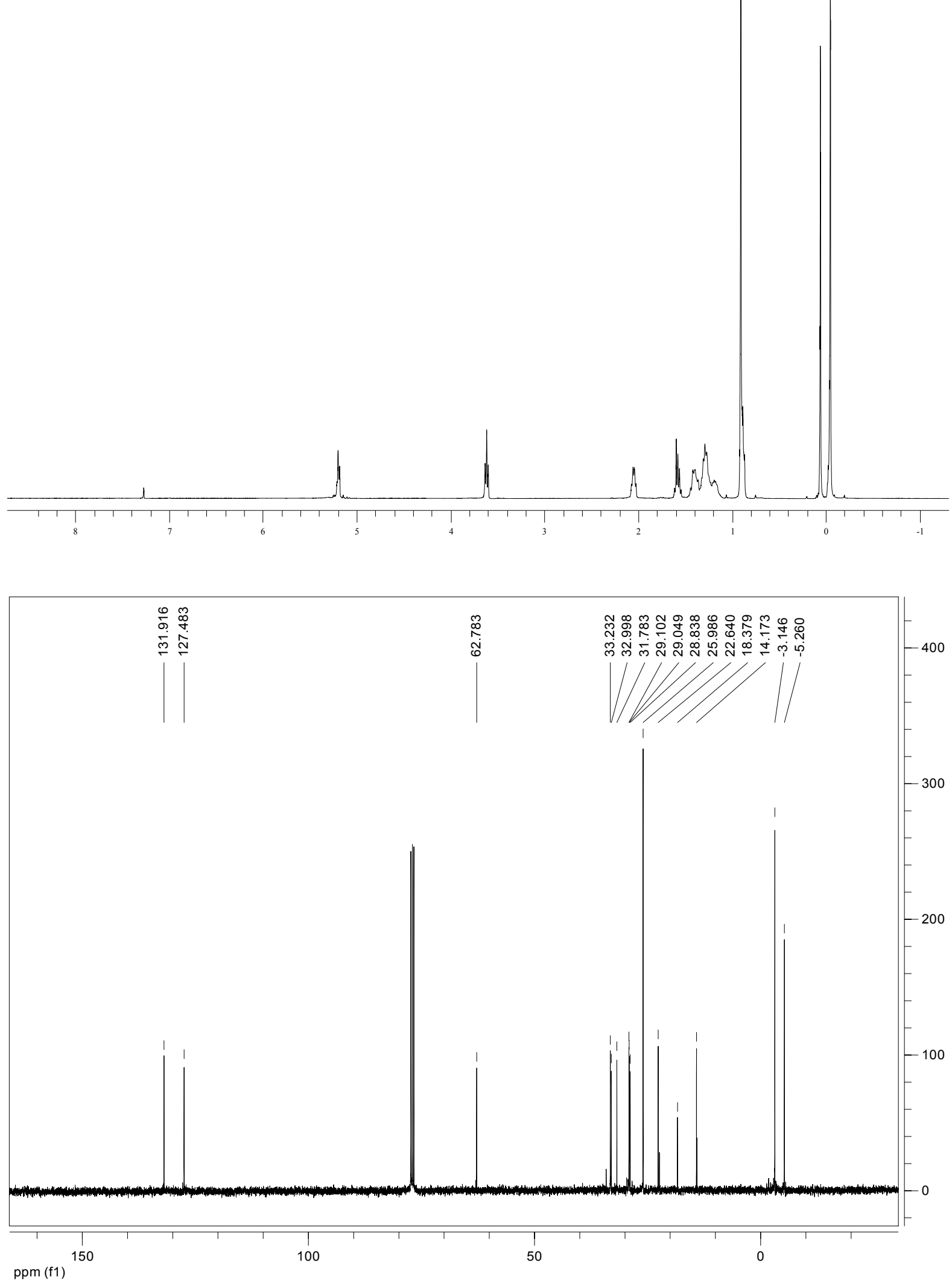
${ }^{1} \mathrm{H}$ and ${ }^{13} \mathrm{C}$ NMR for $6 k$

(N)

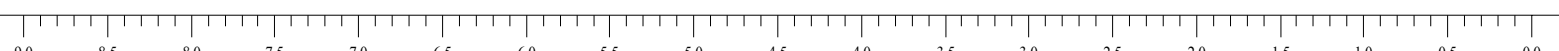

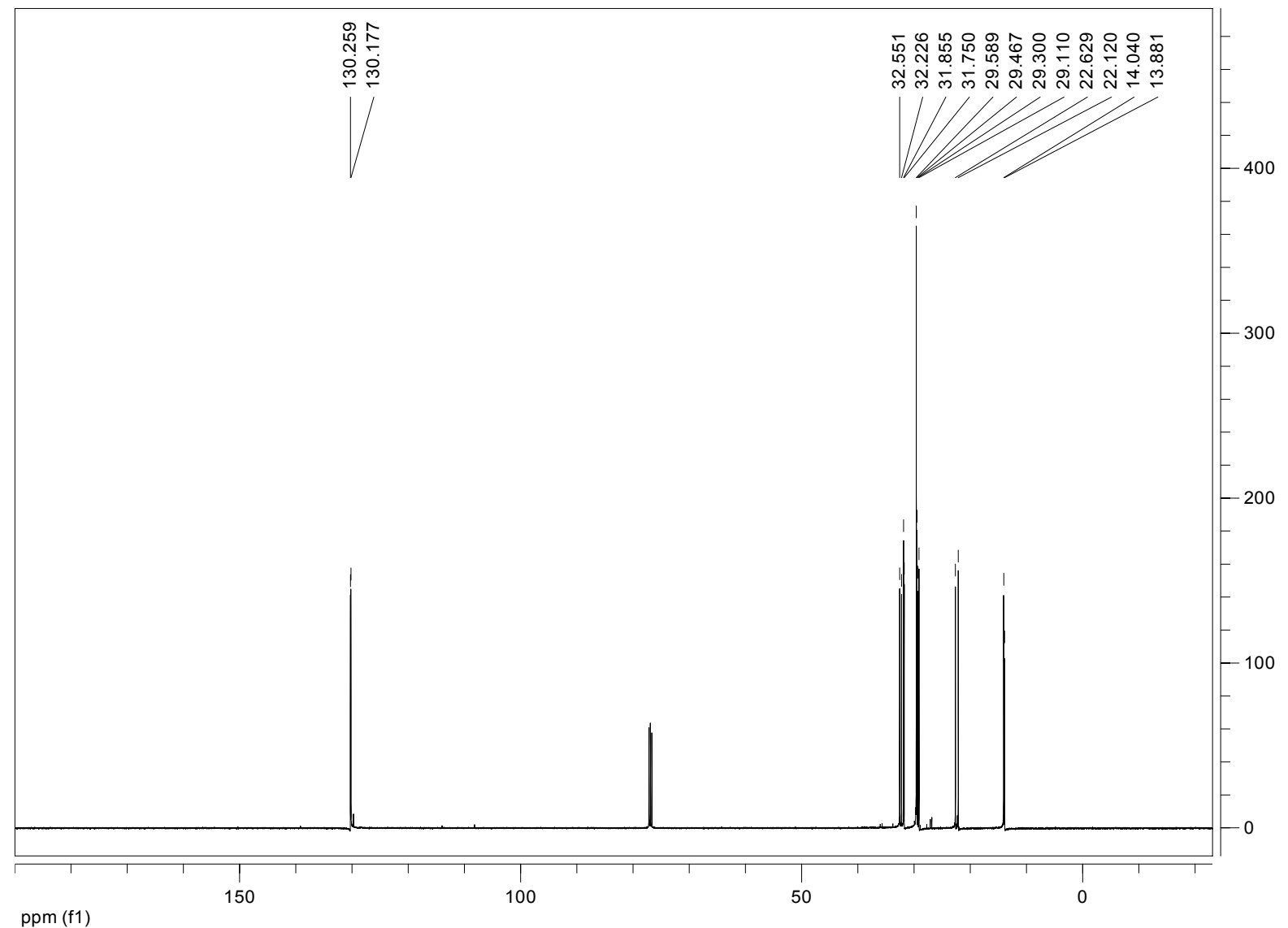




\section{S 36}

${ }^{1} \mathrm{H}$ and ${ }^{13} \mathrm{C}$ NMR for $6 \mathrm{I}$<smiles>C/C=C/CCCCCCCCCC</smiles>

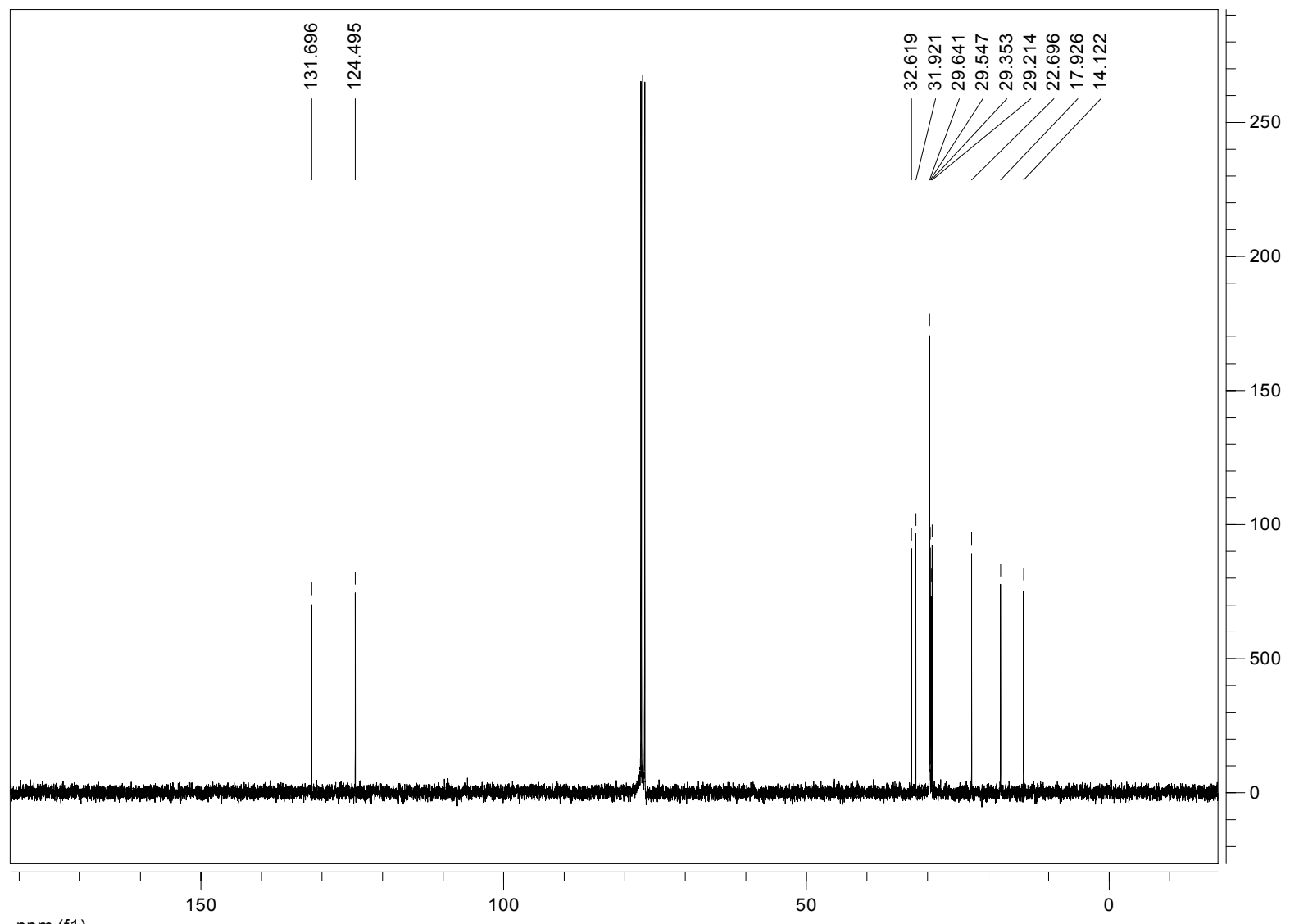

ppm (f1) 
${ }^{1} \mathrm{H}$ and ${ }^{13} \mathrm{C}$ NMR for $9 a$

(1)
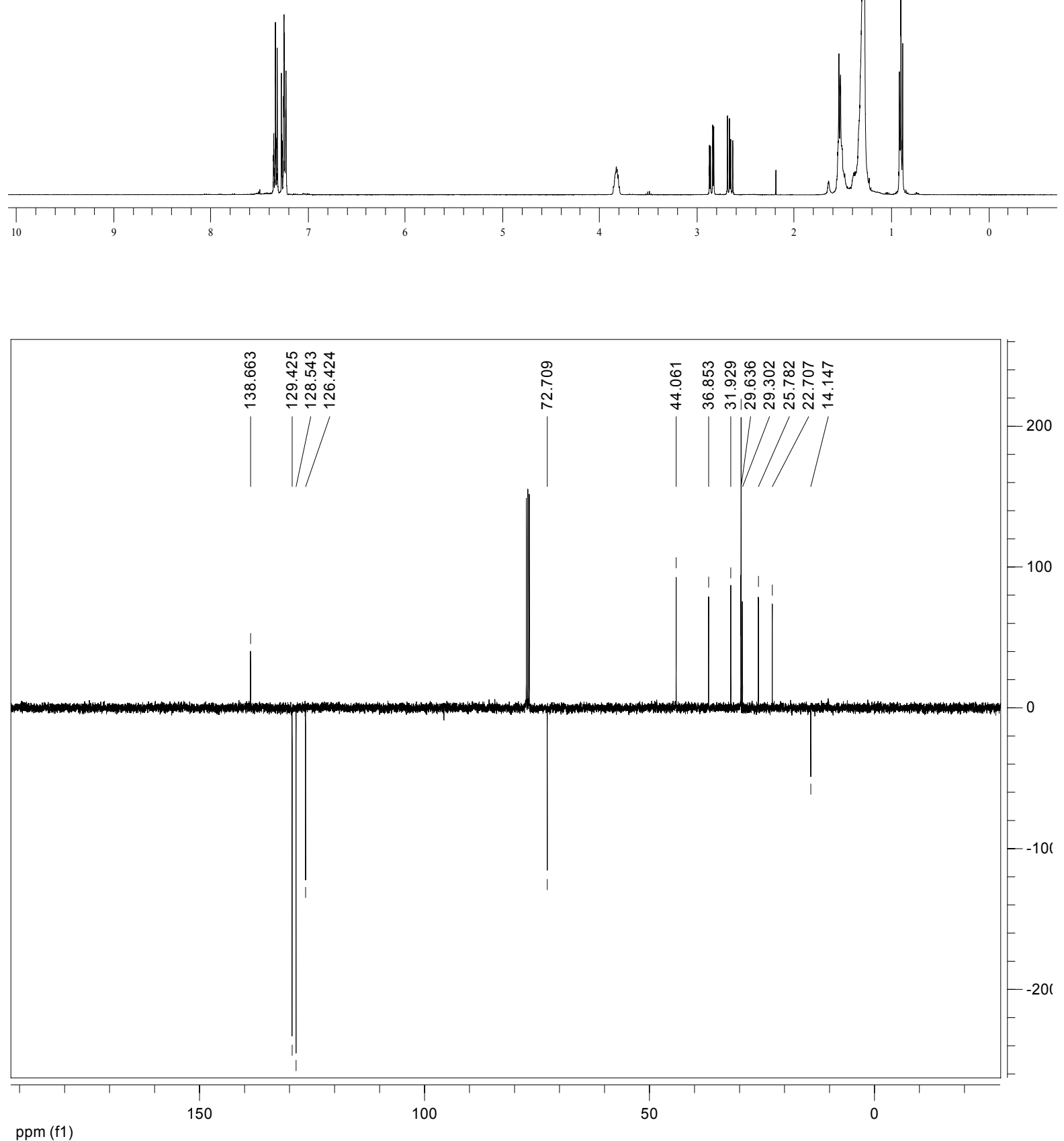


\section{S 38}

${ }^{1} \mathrm{H}$ and ${ }^{13} \mathrm{C}$ NMR for $9 b$

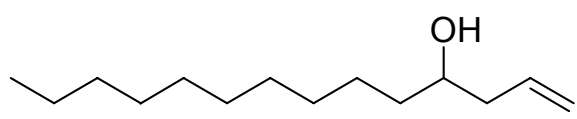
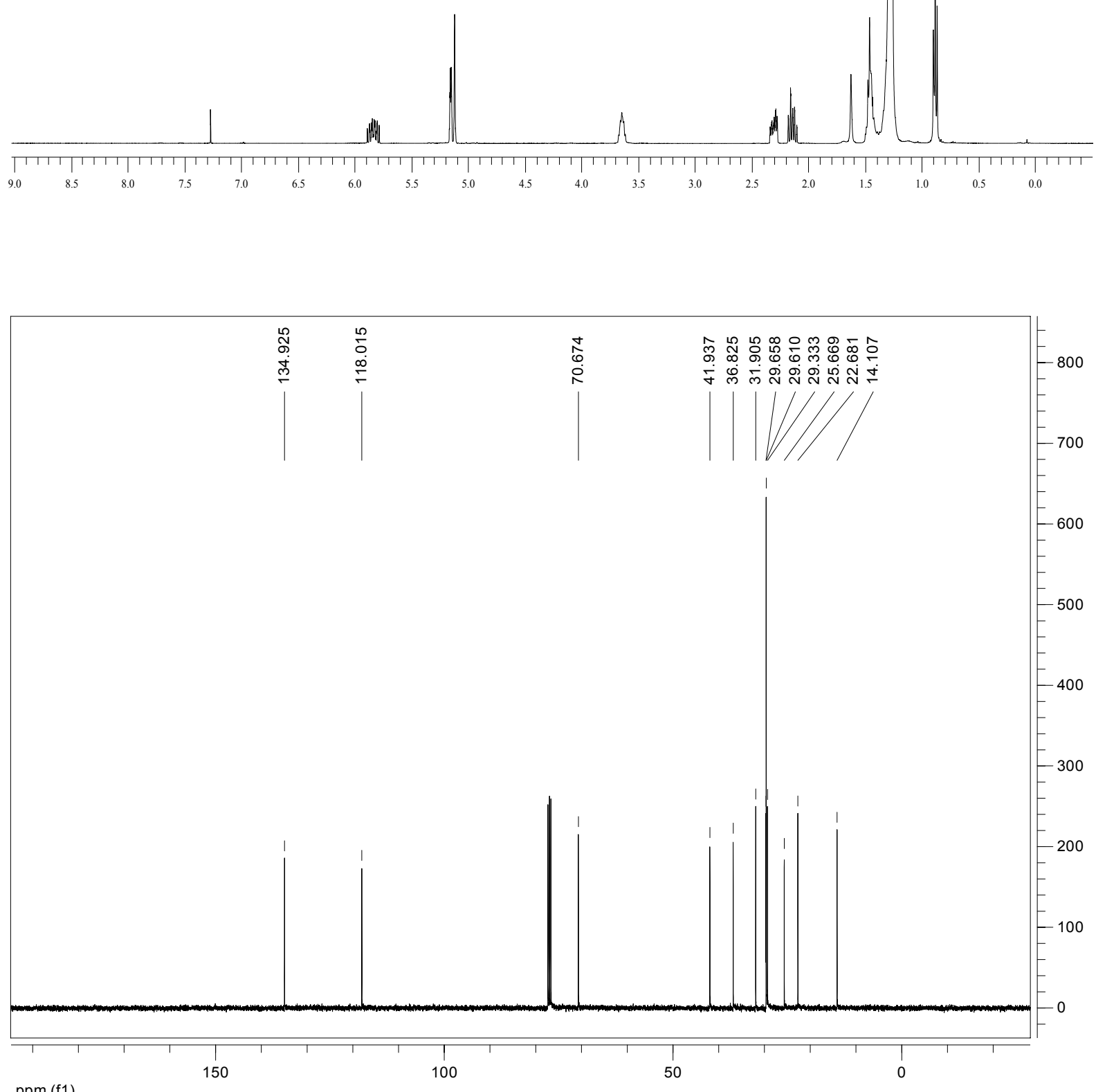

ppm (f1) 
${ }^{1} \mathrm{H}$ and ${ }^{13} \mathrm{C}$ NMR for $9 \mathrm{c}$

$\mathrm{C}^{\mathrm{OH}}$
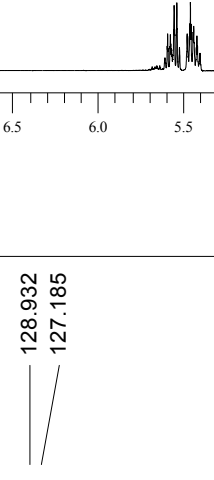

$\underset{\substack{\infty \\ 0}}{\substack{\infty \\:}}$

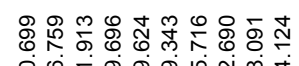

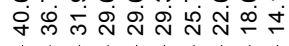

$\stackrel{1}{N}$

H H WIS
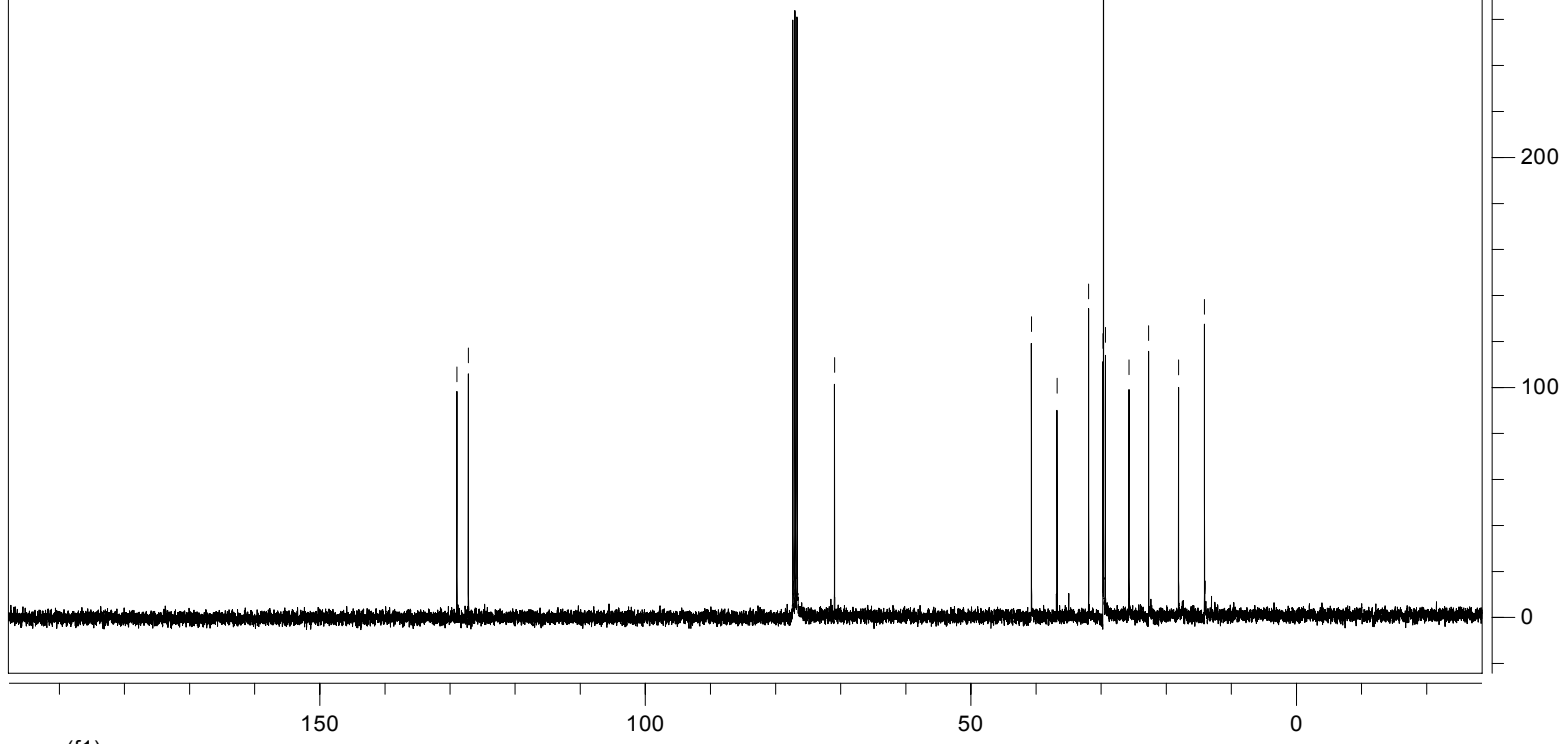

ppm (f1) 


\section{S 40}

${ }^{1} \mathrm{H}$ and ${ }^{13} \mathrm{C}$ NMR for $\mathbf{9 d}$

$\mathrm{C}^{\mathrm{OH}}$

N

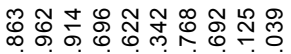

ஸ்
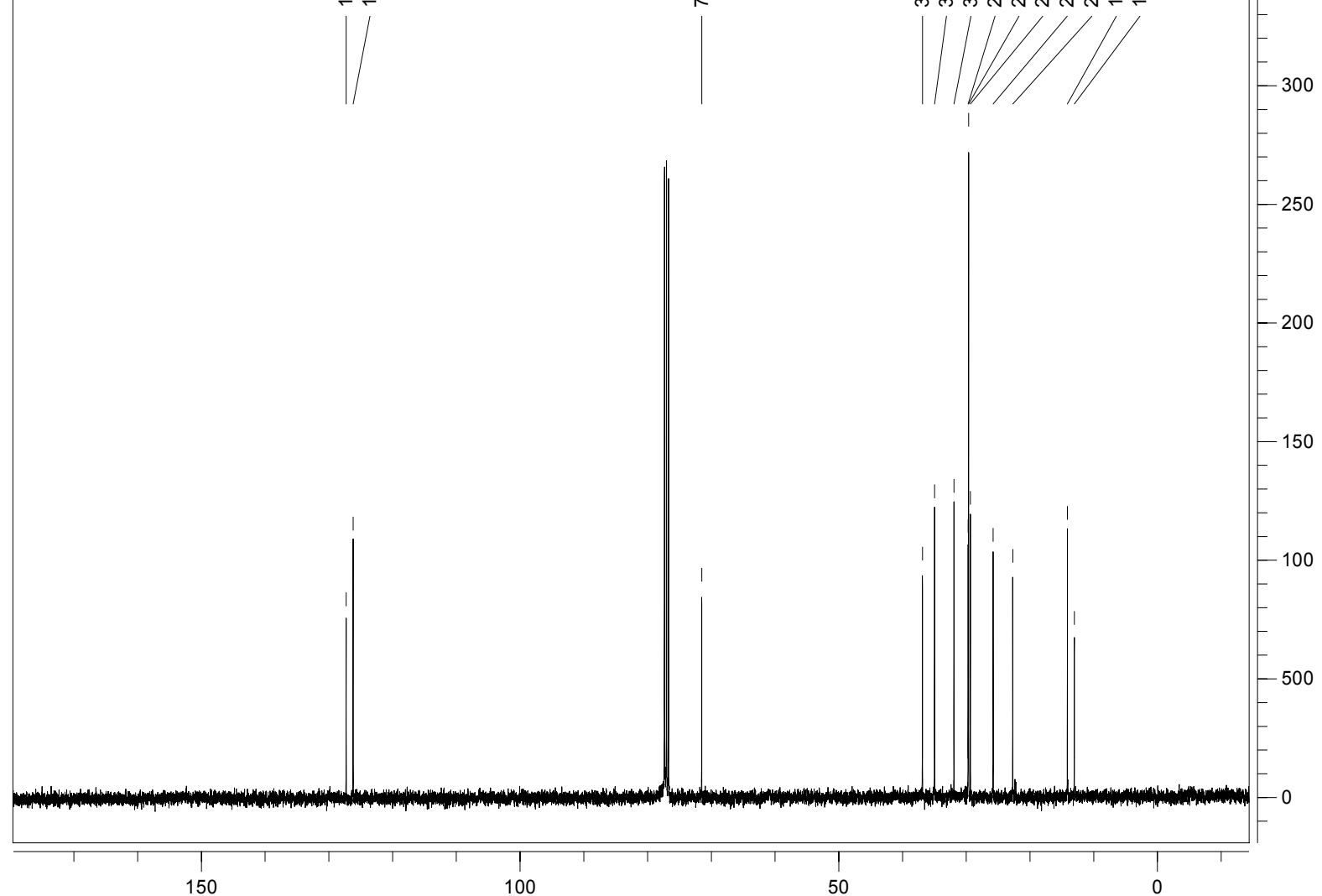

ppm (f1)

100 


\section{S 41}

\section{${ }^{1} \mathrm{H}$ and ${ }^{13} \mathrm{C}$ NMR for $9 e$}<smiles>CCCCCCCCCCC(O)CC[As](C)(=O)=O</smiles>

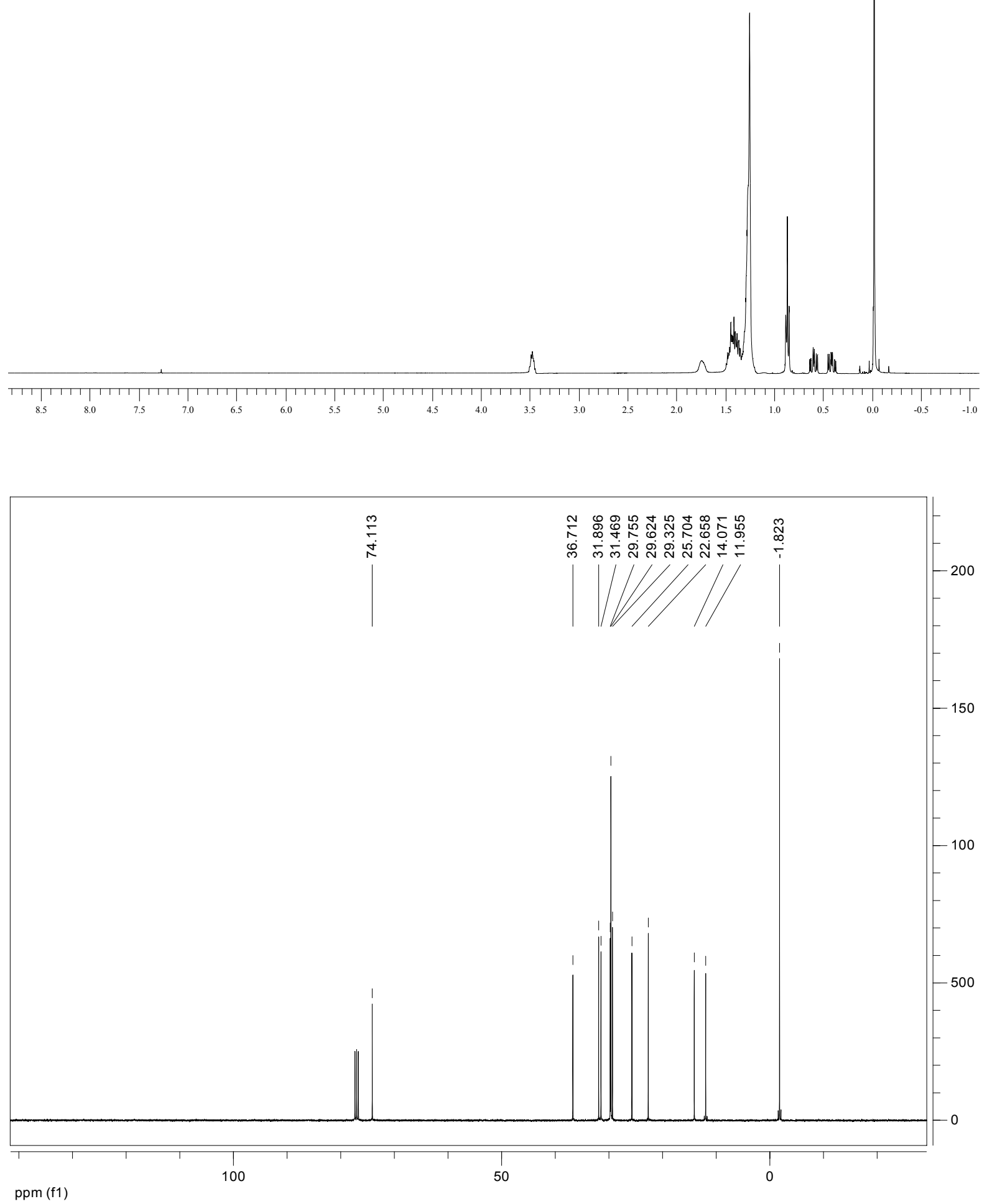


${ }^{1} \mathrm{H}$ and ${ }^{13} \mathrm{C}$ NMR for $9 f$

$\overbrace{}^{C^{T M S}}$
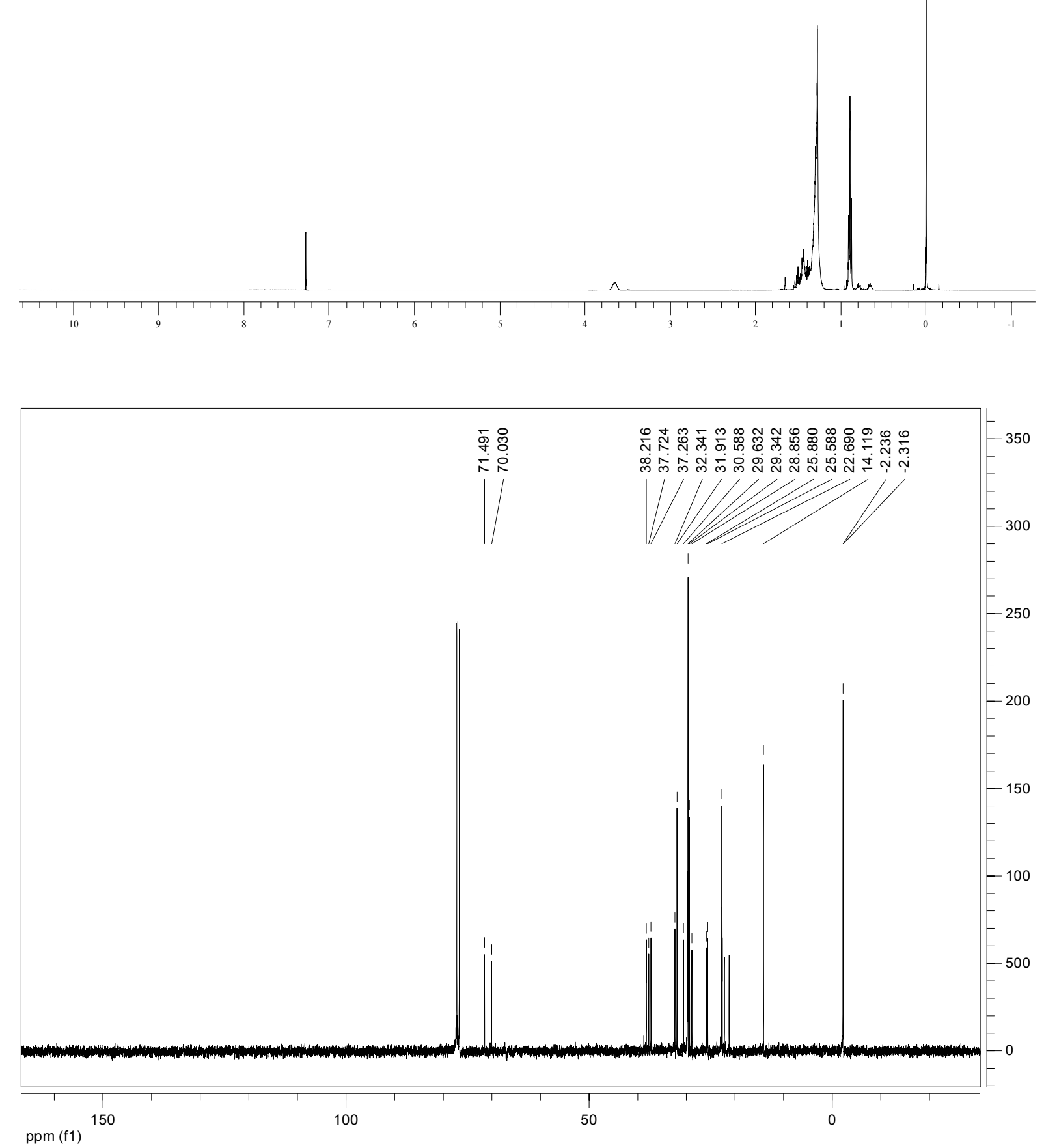